\title{
Improving Power System Stability with Gramian Matrix-Based Optimal Setting of a Single Series FACTS Device: Feasibility Study in Vietnamese Power System
}

\author{
Le Van Dai, ${ }^{1,2}$ Doan Duc Tung, ${ }^{3}$ Tran Le Thang Dong, ${ }^{1}$ and Le Cao Quyen ${ }^{4,5}$ \\ ${ }^{1}$ Duy Tan University, Da Nang 550000, Vietnam \\ ${ }^{2}$ Faculty of Electrical Technology, Inductrial University of Ho Chi Minh City, Ho Chi Minh City 700000, Vietnam \\ ${ }^{3}$ Faculty of Engineering and Technology, Quy Nhon University, Binh Dinh 820000, Vietnam \\ ${ }^{4}$ Department for Management of Science and Technology Development, Ton Duc Thang University, Ho Chi Minh City 700000, Vietnam \\ ${ }^{5}$ Faculty of Electrical \& Electronics Engineering, Ton Duc Thang University, Ho Chi Minh City 700000, Vietnam
}

Correspondence should be addressed to Le Cao Quyen; lecaoquyen@tdt.edu.vn

Received 17 October 2016; Revised 11 November 2016; Accepted 23 November 2016; Published 29 January 2017

Academic Editor: Dimitri Volchenkov

Copyright (C) 2017 Le Van Dai et al. This is an open access article distributed under the Creative Commons Attribution License, which permits unrestricted use, distribution, and reproduction in any medium, provided the original work is properly cited.

\begin{abstract}
The Vietnamese power system has experienced instabilities due to the effect of increase in peak load demand or contingency grid faults; hence, using flexible alternating-current transmission systems (FACTS) devices is a best choice for improving the stability margins. Among the FACTS devices, the thyristor-controlled series capacitor (TCSC) is a series connected FACTS device widely used in power systems. However, in practice, its influence and ability depend on setting. For solving the problem, this paper proposes a relevant method for optimal setting of a single TCSC for the purpose of damping the power system oscillations. This proposed method is developed from the combination between the energy method and Hankel-norm approximation approach based on the controllability Gramian matrix considering the Lyapunov equation to search for a number of feasible locations on the small-signal stability analysis. The transient stability analysis is used to compare and determine appropriate settings through various simulation cases. The effectiveness of the proposed method is confirmed by the simulation results based on the power system simulation engineering (PSS/E) and MATLAB programs. The obtained results show that the proposed method can apply to immediately solve the difficulties encountering in the Vietnamese power system.
\end{abstract}

\section{Introduction}

Motivation. When the social, political, and technological aspects develop, the demand of electric power grows rapidly, resulting in the increase in the scale and complexity of power systems. Some characteristics, such as long transmission distances over weak grids, highly variable generation patterns, and heavy load, tend to increase the wide-area electromechanical oscillations. These oscillations threaten the secure operation of the power systems and if not controlled efficiently can lead to generator outages, line tripping, and even large-scale blackouts.

During over several decades, a number of electrical power systems in the world have been faced with the serious power system blackouts, such as Indian on July 30 and 31, 2012 [1]; Dubai on June 9, 2005; Malaysia on Jan. 13, 2005; Kuwait on Nov. 01, 2004; Australia on Aug. 14, 2004; Shanghai on Aug. 27, 2003; Sweden/Denmark on Sept. 23, 2003; Finland on Aug., 1992; Western France on Jan. 12, 1987; Florida on Jan. 12, 1987; Florida, USA, on May 17, 1985; Belgium on Aug. 4, 1982 [2-5]. Typically, the event is on August 10, 1996, western blackout of Western Systems Coordinating Council (WSCC) interconnection, caused by the negative damping of the $0.25 \mathrm{~Hz}$ western interarea mode [6]. As a result, leading to the split of the system into four large islands, over 7.5 million customers experienced outages ranging from a few minutes to nine hours, and total load loss is $30,500 \mathrm{MW}$ because of the poor oscillations damping. Figures 1(a) and 1(b) show 


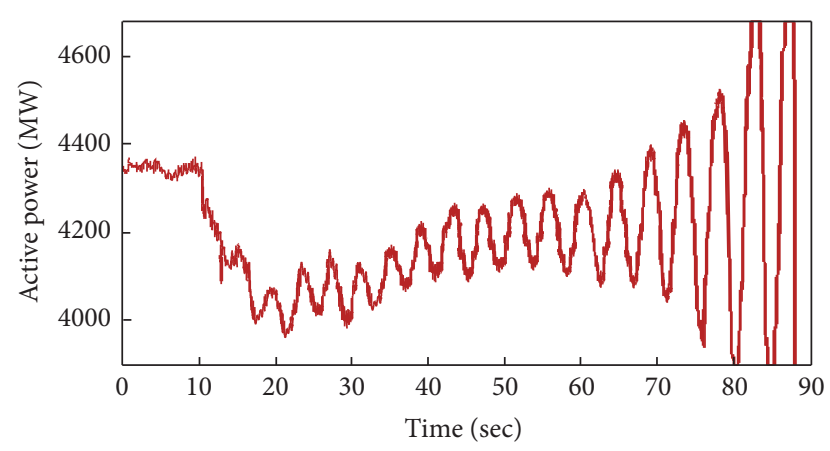

(a)

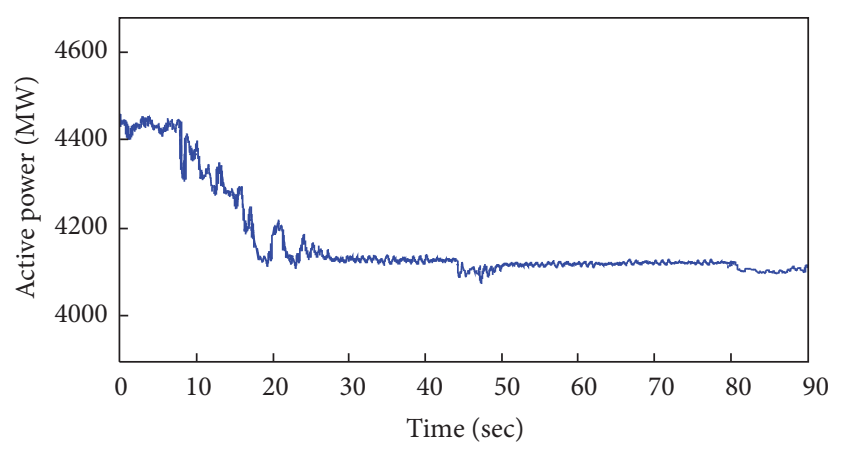

(b)

FIGURE 1: The event of the small-signal instability during the blackout WSCC system on Aug. 10, 1996: (a) the observed California Oregon Interconnections power (Dittmer control center); (b) the simulated California Oregon Interconnections power (initial base case).

the measured and simulated power swings of this event. The measured data show that the standard planning models could be unreliable predictors of oscillatory behavior.

In recent years, the economic tempo in the Vietnam has been developed rapidly; the total load has increased continuously. Accordingly, the Vietnamese power system has been faced with the serious power system blackouts, such as on Dec. 27, 2006; July 20, 2007; Apr. 09, 2007; and the latest event on May 22, 2013. All of the technical problems that the Vietnamese power system identified after these events had already been reported $[7,8]$.

The power system oscillations occur in the power systems because of the contingencies, such as the grid faults and sudden load changes; the dampening of these oscillations is necessary for a secure system operation. If the controlled systems react quickly against faults, the power system stability will enhance significantly. The advanced power electronics has led to a new design called flexible alternating-current transmission systems (FACTS) by Electrical Power Research Institute (EPRI). The FACTS devices make more use of the exiting capacities in the power system and enhance the power system stability. For example, the parameters in the power system are controlled and the load flow is modified to preclude the overload of transmission lines after the grid faults. The FACTS devices are widely used to improve the efficiency of power system operation. However, the benefits derived from FACTS controllers, such as the small-signal stability and transient stability that depend greatly on their optimal placement in the power systems $[9,10]$. Therefore, looking for the optimal placement of FACTS devices in the large-scale power systems is an interesting research topic.

State of the Science. The damping of electromechanical oscillations in the power systems is a matter to belong with power engineers and become one of the most interesting research topics [11, 12]. The PSSs are a profound influence solution on improving the power system stability. This solution is based on the excitation system and the generator speed deviation signals, in which the speed deviation is used as the supplementary control signals in order to provide to the automatic voltage regulators. The fast progress in the field of power electronics had contributed to the development of the electrical power industry based on the controllable utilization of FACTS [13]. This device can dampen both the local- and interarea oscillations [10], whereas PSSs could only dampen the local-area oscillations [14]. Thus, FACTS is the best choice to enhance the stability margin of the existing power systems $[9,15]$ and especially to TCSC. This device is considered as one of the most effective FACTS and is widely used to regulate the power flow in transmission lines, dampen the power oscillations, mitigate subsynchronous resonance (SSR), enhance power system stability, and so forth by changing the reactance of transmission line [16]. There is hard sledding for the researchers regarding how to determine optimal location of FACTS.

The methods for solving location problems can be classified into two categories: (i) analytical techniques and (ii) heuristic optimization approaches [17-19]. Among heuristics, Particle Swarm Optimization (PSO) has some advantages as less control parameters, no require preconditions (continuity or differentiability of objective functions), and slow computational burden, which make it popular to solve optimal location and design problems of FACTS [20-24]. However, PSO had drawback as slow convergence in search stage and limit of local search ability; furthermore, the algorithm will not work out the problems of scattering and optimization [15]. Genetic algorithm (GA) has been proposed to solve optimal location and design problems of FACTS $[9,15,19,25]$. This method has been applied to obtain promising results. However, downside of GA is that the requested run time is very long when studying the large-scale systems [26]. In [24], optimal location of TCSC is determined via PSO whose objective is to maximize small-signal stability. In $[9,23]$, a mathematical objective function is used to derive objective function for GA and PSO by considering the load-ability in the system security margins, respectively. In [27], the authors used a method based on hybrid between PSO and GA for optimal allocation of TCSC to enhance voltage stability and reduce power system losses. The brainstorm optimization algorithm (BSOA) is new heuristic optimization algorithm proposed by the authors in [28] to seek the optimal location of 
TCSC for enhancing the voltage profile. The obtained results are better than PSO and GA.

For the analytical approaches, the modal controllability index has been developed by the authors in [29] to find suitable location of TCSC for dampening interarea model of oscillations. However, the authors consider the simulation with or without FACTS placed in the power system to calculate the maximum controllability index values corresponding to critical mode. Particularly, their main interest is in the input signals that do not know what is occurring at output of FACTS through observability index values. In [30], Vaidya and Rajderkar used the sensitivity-based method and the authors in [31] applied real power flow performance index sensitivity to determine the optimal location of TCSC to enhance the power system stability. In [32], the eigenvalue sensitivity method is utilized to find optimal location of controllable series capacitors for dampening power system location. The eigenvalue sensitivity values are calculated based on the modal controllability and observability indices of series reactance modulation. In [33], the suitable location of series compensators is determined based on trajectory sensitivity analysis. The objective is to maximize the benefit of series compensators in order to improve the generator rotor angle stability. The energy method based on Gramian matrices is another technique developed by the authors in $[34,35]$ to determine the optimal setting of TCSC and static VAR compensator (SVC) obtained with promising result. However, the difficult of this method is when calculating the too large-scale power systems.

It is observed that most of existing methods in the previous literatures have been proposed recently for the location of FACTS. These methods have several drawbacks; firstly, the computation of critical modes may be questionable in case of large-scale power system since they may not be unique. Moreover, the computation of them also depends on the local or interarea modes. Secondly, the computation of participation factors is only based on the state variables and neglects the input-output behavior. Thirdly, it just focuses on analyzing the small-scale power systems. Therefore, in order to overcome these drawbacks, this paper is a continuation of [34] and combines the Hankel-norm approximation method to determine the best location for installing TCSC with objective for damping power system oscillation of the practical power system, to wit Vietnamese power system. It indicates that using the Gramian matrix-based method to calculate the complexity and large-scale power systems takes a lot of time since the system state matrix is very large. Therefore, the Hankel-norm approximation method [36] is proposed to solve such problem. The selection of the input signal for TCSC controller is an important consideration for seeking the optimal location to dampen the interarea oscillations, in which the line reactive and active power, line current, and bus voltage are all good selections. In this study, the active power in transmission line is considered as an effective input signal for TCSC controller. The contingency cases are considered based on the active power perturbation signals in the transmission lines that were selected on the basis of the real power line flow performance index (PI) introduced in [37]. In addition, in order to determine the parameters of the TCSC controller, the optimal method in [38] is also considered in this paper.

Contribution. In this paper, a relevant method for determining the optimal placement of TCSC controller is proposed to enhance the stability of large-scale power systems. This proposed method is developed from the energy approach based on the controllability Gramian matrix of the linearized system. The multimachine power system with TCSC controller is expressed in the form of a differential algebraic equation (DAE) model. The controllability Gramian matrix is obtained from the unique positive definite solutions of the Lyapunov equation and it depends on the control inputoutput and state matrixes of system. The optimal location is selected based on the maximum total Gramian energy calculated from the contingency outage cases (disturbance in lines) on the small-signal stability analysis that means the control input must insure the smallest control energy. In line with this purpose, the Hankel-norm approximation method is applied to reduce the number of state variables when dealing with large-scale power systems.

The main new contributions of this paper are summarized as follows:

(i) To develop a relevant method to determine the optimal location of FACTS on the small-signal stability analysis

(ii) To propose an association between proposed method and the Hankel-norm approximation method to limit the time calculation, so that the proposed method can be easily implemented for complexity and large-scale power systems.

The remainder of this paper is organized as follows: Section 2 addresses the principle of characteristics of the Vietnamese power system and the differential algebraic equation (DAE) model of the power system with and without TCSC controller. The proposed method for optimal location of TCSC controller based on the Gramian matrices and the Hankel-norm approximation method are introduced in Section 3. The case studies and conclusions are given in Sections 4 and 5, respectively. Finally, the algorithm for the transformation matrix can be found in the Appendix.

\section{Theoretical Analysis}

2.1. Characteristics of the Vietnamese Power System. The Vietnamese $500 \mathrm{kV}$ power system operated in 1994 . The length of transmission line of about $1,500 \mathrm{~km}$ was connected from the HoaBinh to PhuLam power stations with total rating of 2,700 MVA. It reached the length of about $5690 \mathrm{~km}$ and total capacity of all the $500 \mathrm{kV}$ substations of 22,800 MVA in 2015. Several areas of $500 \mathrm{kV}$ transmission lines are compensated by shunt reactors of about $70 \%$ and series capacitors of about $60 \%$. The total generation capacity of the Vietnamese power system could reach $60,000 \mathrm{MW}$ by the end of 2020 [39]. The result of load flow calculation on the $500 \mathrm{kV}$ power system is shown in Figure 2. 


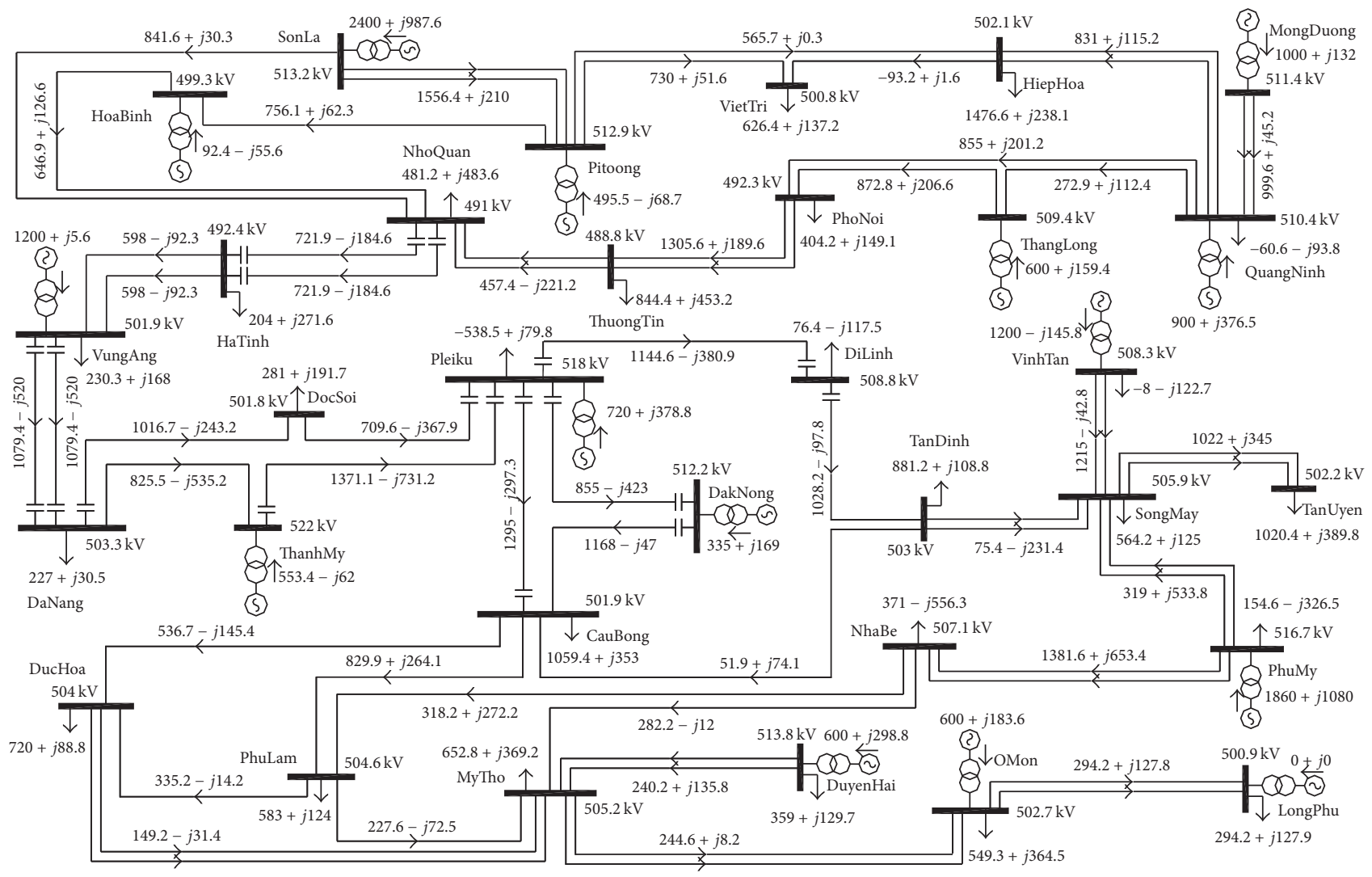

FIGURE 2: The result of load flow calculation on the Vietnamese $500 / 220 \mathrm{kV}$ power system 2020.

2.2. DAE Model of Power System. The methodological method for dynamic modeling of general $m$-machine and $n$-bus power system has been described in [40] and is applied for this work. In this model, each synchronous generator is represented by two-axis flux decay dynamic model along with IEEE type I. The differential algebraic equation (DAE) model of the power system without TCSC controller can be expressed as follows:

$$
\begin{array}{cc}
x_{\text {sys }}=f\left(x_{\text {sys }}, y_{\text {sys }}, u_{\text {sys }}\right) & x_{\text {sys }}(0)=x_{0} \\
0=g\left(x_{\text {sys }}, y_{\text {sys }}, u_{\text {sys }}\right) & y_{\text {sys }}(0)=y_{0}
\end{array}
$$

in which $x_{\text {sys }}, y_{\text {sys }}$, and $u_{\text {sys }}$ are, respectively, the state, algebraic, and input vectors and are defined as

$$
\begin{aligned}
& x_{\text {sys }}= {\left[\delta_{i}, \omega_{i}, E_{q i}^{\prime}, E_{d i}^{\prime}, E_{f d i}, V_{R i}, R_{F i}\right]^{\mathrm{T}}, } \\
& y_{\text {sys }}= {\left[V_{j}, \theta_{j}, I_{d i}, I_{q i}\right]^{\mathrm{T}}, } \\
& u_{\text {sys }}= {\left[T_{M i}, V_{\mathrm{REFi}}\right]^{\mathrm{T}}, } \\
& \quad i=1, \ldots, m ; j=1, \ldots, n,
\end{aligned}
$$

where

$\mathrm{T}$ is the transpose operator,

$\delta$ is the rotor angle of generator, $\omega$ is the speed of generator,

$V$ is the voltage magnitude of bus,

$\theta$ is the power angle of bus,

$I_{d}$ are the $d$-axis components of the current of generator,

$I_{q}$ are the $q$-axis components of the current of generator,

$V_{R}$ is the input amplifier voltage of the excitation of generator,

$R_{F}$ is the stabilizer feedback variable of the excitation of generator,

$T_{M}$ is the electrical power of generator,

$E_{q}^{\prime}$ is the $q$-axis component of the internal voltage of generator,

$E_{d}^{\prime}$ is the $d$-axis component of the internal voltage of generator,

$E_{f d}$ is the $d$-axis component of the field voltages of generator,

$V_{\mathrm{REF}}$ is the reference voltage of generator. 


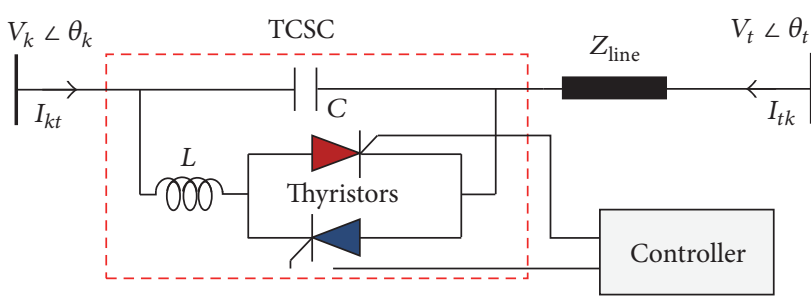

(a)

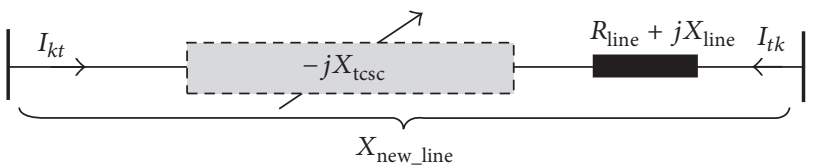

(b)

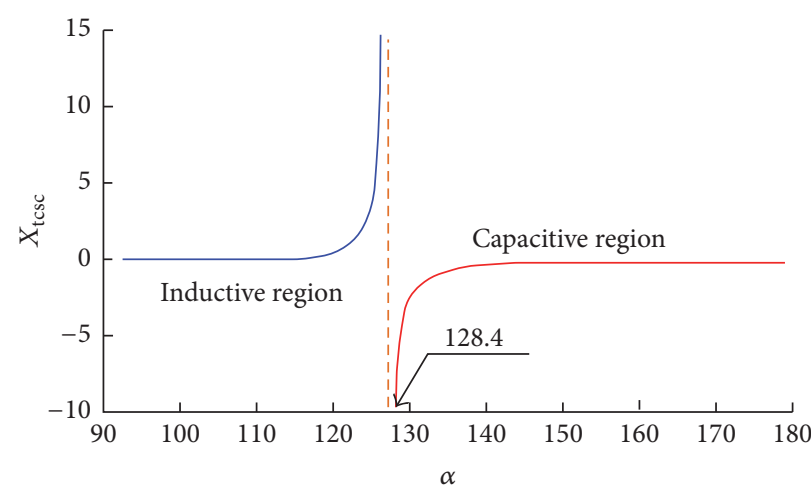

(c)

FIGURE 3: The TCSC controller: (a) structure; (b) equivalent; (c) reactance versus firing angle characteristic cure.

Next, the linearized model is given as [40]

$$
\begin{aligned}
{\left[\begin{array}{c}
\Delta \dot{X}_{\text {sys }} \\
0 \\
0
\end{array}\right]=} & {\left[\begin{array}{ccc}
A_{\text {sys }}^{\prime} & B_{\text {sys1 }}^{\prime} & B_{\text {sys2 }}^{\prime} \\
C_{\text {sys1 }}^{\prime} & D_{\text {sys11 }}^{\prime} & D_{\text {sys12 }}^{\prime} \\
C_{\text {sys2 }}^{\prime} & D_{\text {sys } 21}^{\prime} & D_{\text {sys22 }}^{\prime}
\end{array}\right]\left[\begin{array}{c}
\Delta X_{\text {sys }} \\
\Delta y_{\text {sys } 1} \\
\Delta y_{\text {sys } 2}
\end{array}\right] } \\
& +\left[\begin{array}{c}
E_{\text {sys }} \\
0 \\
0
\end{array}\right] \Delta U_{\text {sys. }}
\end{aligned}
$$

It can be identified as

$$
\begin{aligned}
A_{\text {sys }} & =A_{\text {sys' }}^{\prime} \\
B_{\text {sys }} & =\left[\begin{array}{ll}
B_{\text {sys1 }}^{\prime} & B_{\text {sys } 2}^{\prime}
\end{array}\right], \\
C_{\text {sys }} & =\left[\begin{array}{l}
C_{\text {sys1 }}^{\prime} \\
C_{\text {sys2 }}^{\prime}
\end{array}\right], \\
D_{\text {sys }}= & {\left[\begin{array}{ll}
D_{\text {sys } 11}^{\prime} & D_{\text {sys12 }}^{\prime} \\
D_{\text {sys } 21}^{\prime} & D_{\text {sys } 22}^{\prime}
\end{array}\right], } \\
E_{\text {sys }}= & {\left[\begin{array}{c}
E_{\text {sys } 1} \\
0 \\
0
\end{array}\right] . }
\end{aligned}
$$
[40]:

Equation (3) can be changed to another form as follows

$$
\begin{aligned}
\Delta \dot{x}_{\text {sys }} & =A_{\text {sys }} \Delta x_{\text {sys }}+B_{\text {sys }} \Delta y_{\text {sys }}+E_{\text {sys }} \Delta u_{\text {sys }}, \\
0 & =C_{\text {sys }} \Delta x_{\text {sys }}+D_{\text {sys }} \Delta y_{\text {sys }}
\end{aligned}
$$

where

$$
\begin{aligned}
& \Delta x_{\text {sys }}=\left[\Delta \delta_{i}, \Delta \omega_{i}, \Delta E_{q i}^{\prime}, \Delta E_{d i}^{\prime}, \Delta E_{f d i}, \Delta V_{R i}, \Delta R_{F i}\right]^{\mathrm{T}} \text {, } \\
& \Delta y_{\mathrm{sys}}=\left[\Delta y_{\mathrm{sys} 1}, \Delta y_{\mathrm{sys} 1}\right] \\
& =\left[\begin{array}{llllll}
\theta_{1} & V_{1} \cdots V_{m} & \theta_{2} \cdots \theta_{n} & V_{m+1} & \cdots V_{n}
\end{array}\right]^{\mathrm{T}}, \\
& \Delta u_{\text {sys }}=\left[\Delta T_{M i}, \Delta V_{\mathrm{REF} i}\right]^{\mathrm{T}} \text {. }
\end{aligned}
$$

\subsection{DAE Model of Power System with TCSC Controller}

2.3.1. TCSC Controller. The main role of TCSC is to control fast the active power flow, increase the power transfer on transmission line, and enhance the stability of the power system. The basic structure TCSC consists of a fixed series capacitor bank $\mathrm{C}$ in parallel with a thyristor-controlled reactor (TCR), as shown in Figure 3(a). It can control the continuous power flow on the alternating-current (AC) line with a variable series capacitive reactance. This series reactance is adjusted through variation of firing angle $\alpha$; the effective reactance $X_{\text {tcsc }}$ depends on three regions: (i) inductive region, which starts increasing from $X_{L} / X_{C}$ value to infinity $\left(X_{L}(\alpha)=X_{C}\right)$; (ii) capacitive region, which starts increasing from infinity to capacitive reactance $X_{C}$; and (iii) resonance region, which occurs between these two regions. In order to avoid the resonance region, the steady state limits of the firing angle are chosen to be $90^{\circ} \leq \alpha \leq 180^{\circ}$ with the resonant point $\alpha_{r}=128.4^{\circ}$. In this paper, for investigating power system stability after a fault, TCSC should operate in capacitive region and the limit of the steady state to the firing angle is chosen to be $\alpha_{r} \leq \alpha \leq 180^{\circ}$, as shown in Figure 3(c). 


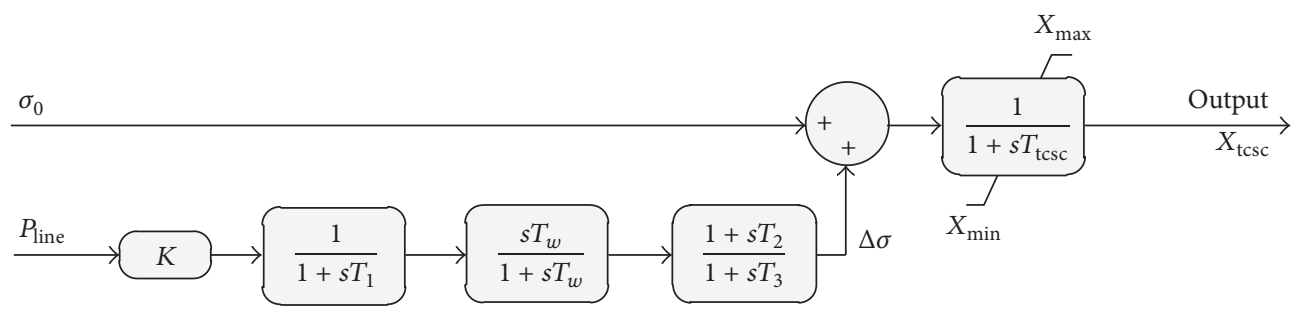

FIGURE 4: The transfer function mode of TCSC controller.

The variable $X_{\text {tcsc }}$ can be obtained by using some of the control strategies and the feedback signal of the TCSC controller. This feedback signal can be the signal of active power, reactive power, current of transmission line, or transmission angle [41]. In this paper, the main objective is to dampen the power oscillations. The active power signal in transmission line is chosen [42] and the control strategy for TCSC is shown in Figure 4 [34].

The new equivalent impedance of the line, when placed TCSC, as shown in Figure 3(b), can be obtained as [43]

$$
X_{\text {new_line }}=X_{\text {line }}+X_{\text {tcsc }}
$$

in which the relationship between the firing angle $\alpha$ and the impedance of the TCSC at fundamental frequency can be derived as follows [44]:

$$
\begin{aligned}
& X_{\mathrm{tcsc}}=-X_{C}+K_{1}(2 \sigma+\sin 2 \sigma) \\
&-K_{2} \cos ^{2} \sigma(\omega \tan (\omega \sigma)-\tan \sigma) \\
& \sigma=\pi-\alpha, \omega=\sqrt{\frac{X_{C}}{X_{L}}}, X_{L C}=\frac{X_{C} X_{L}}{X_{C}-X_{L}}, K_{1} \\
&=\frac{X_{C}+X_{L C}}{\pi}, K_{2}=\frac{4 X_{L C}^{2}}{\pi X_{L}},
\end{aligned}
$$

where $\sigma$ is the conduction angle, $\omega$ is the TCSC ratio. $X_{L}=\omega L$ is the reactance of the inductor, and $X_{L}=1 / \omega C$ is the fixed capacitive impedance.

2.3.2. The Unification of TCSC Controller in Power System. The TCSC has been instated on the transmission line between bases $k$ and $t$ of an $n$-bus power system. The injected power flow into buses $k$ and $t$ is given ib the following equations [45].

At bus $k$,

$$
\begin{aligned}
P_{k} & =\Delta G_{k t}\left|V_{k}\right|^{2}-\left|V_{k}\right|\left|V_{t}\right| \\
& \cdot\left[\Delta G_{k t} \cos \left(\theta_{k}-\theta_{t}\right)+\Delta B_{k t} \sin \left(\theta_{k}-\theta_{t}\right)\right],
\end{aligned}
$$

$$
\begin{aligned}
Q_{k} & =-\Delta B_{k t}\left|V_{k}\right|^{2}-\left|V_{k}\right|\left|V_{t}\right| \\
\cdot & {\left[\Delta G_{k t} \sin \left(\theta_{k}-\theta_{t}\right)-\Delta B_{k t} \cos \left(\theta_{k}-\theta_{t}\right)\right] . }
\end{aligned}
$$

Similarly, at bus $t$,

$$
\begin{aligned}
P_{t} & =\left|V_{t}\right|^{2} \Delta G_{k t}-\left|V_{k}\right|\left|V_{t}\right| \\
\cdot & {\left[\Delta G_{t k} \cos \left(\theta_{k}-\theta_{t}\right)+\Delta B_{t k} \sin \left(\theta_{k}-\theta_{t}\right)\right] } \\
Q_{t} & =-\left|V_{t}\right|^{2} \Delta B_{k t}+\left|V_{k}\right|\left|V_{t}\right| \\
\cdot & {\left[\Delta G_{k t} \sin \left(\theta_{k}-\theta_{t}\right)-\Delta B_{t k} \cos \left(\theta_{k}-\theta_{t}\right)\right], }
\end{aligned}
$$

where $P_{k}, Q_{k}, P_{t}$, and $Q_{t}$ are the active and reactive powers injected at buses $k$ and $t$, respectively. Also, $V_{k}, \theta_{k}, V_{t}$, and $\theta_{t}$ are voltage magnitudes and phase angles of buses $k$ and $t$, respectively. $\Delta G$ and $\Delta B$ depend on TCSC reactance and are given as

$$
\begin{aligned}
& \Delta G_{t k}=\frac{X_{\mathrm{tcsc}} R_{\text {line }}\left(X_{\mathrm{tcsc}}-2 X_{\text {line }}\right)}{\left(R_{\text {line }}^{2}+X_{\text {line }}^{2}\right)\left[R_{\text {line }}^{2}+\left(X_{\mathrm{tcsc}}-X_{\text {line }}\right)^{2}\right]}, \\
& \Delta B_{k t}=\frac{X_{\mathrm{tcsc}}\left[R_{\text {line }}^{2}-X_{\text {line }}^{2}+X_{\mathrm{tcsc}} X_{\text {line }}\right]}{\left(R_{\text {line }}^{2}+X_{\text {line }}^{2}\right)\left[R_{\text {line }}^{2}+\left(X_{\text {tcsc }}-X_{\text {line }}\right)^{2}\right]},
\end{aligned}
$$

where $R_{\text {line }}$ and $X_{\text {line }}$ represent the resistance and reactance of the line, respectively, and $X_{\text {tcsc }}$ is the optimal value of TCSC. The linearized model of TCSC is given as follows:

$$
\begin{gathered}
\Delta \dot{X}_{\mathrm{tcsc}}=A_{\mathrm{tcsc}} \Delta X_{\mathrm{tcsc}}+B_{\mathrm{tcsc}}\left[\Delta \theta_{k}, \Delta \mathrm{V}_{k}, \Delta \theta_{t}, \Delta V_{t}\right]^{\mathrm{T}}, \\
{\left[\Delta P_{k}, \Delta Q_{k}, \Delta P_{t}, \Delta Q_{t}\right]^{\mathrm{T}}} \\
\quad=C_{\mathrm{tcsc}} \Delta X_{\mathrm{tcsc}}+D_{\mathrm{tcsc}}\left[\Delta \theta_{k}, \Delta V_{k}, \Delta \theta_{t}, \Delta V_{t}\right]^{\mathrm{T}} .
\end{gathered}
$$


Incorporating (3) and (13), the DAE model of the multimachine power system with TCSC controller can be described as follows:

$$
\begin{aligned}
& {\left[\begin{array}{c}
\Delta \dot{X}_{\text {sys }} \\
\Delta \dot{X}_{\text {tcsc }} \\
0 \\
0
\end{array}\right]} \\
& =\left[\begin{array}{cccc}
A_{\text {sys }}^{\prime} & A_{1 \mathrm{tcsc}} & B_{\text {sys1 }}^{\prime} & B_{\text {sys2 }}^{\prime} \\
A_{2 \mathrm{tcsc}} & A_{\mathrm{tcsc}} & B_{1 \mathrm{tcsc}}^{\prime} & B_{2 \mathrm{tcsc}}^{\prime} \\
C_{\text {sys }}^{\prime} & C_{1 \mathrm{tcsc}} & D_{\text {sys11 }}^{\prime} & D_{\text {sys12 }}^{\prime} \\
C_{\text {sys2 }}^{\prime} & C_{2 \mathrm{tcsc}} & D_{1 \mathrm{tcsc}}^{\prime} & D_{2 \mathrm{tcsc}}^{\prime}
\end{array}\right]\left[\begin{array}{c}
\Delta X_{\text {sys }} \\
\Delta X_{\mathrm{tcsc}} \\
\Delta y_{\text {sys } 1} \\
\Delta y_{\text {sys } 2}
\end{array}\right] \\
& +\left[\begin{array}{c}
E_{\text {sys-tcsc }} \\
0 \\
0 \\
0
\end{array}\right] \Delta U_{\text {sys-tcsc }}
\end{aligned}
$$

It can be identified as

$$
\begin{aligned}
A_{\text {new }} & =\left[\begin{array}{ll}
A_{\text {sys }}^{\prime} & A_{1 \mathrm{tcsc}} \\
A_{2 \mathrm{tcsc}} & A_{\mathrm{tcsc}}
\end{array}\right], \\
B_{\text {new }} & =\left[\begin{array}{ll}
B_{\text {sys1 }}^{\prime} & B_{\text {sys } 2}^{\prime} \\
B_{\mathrm{tcsc}}^{\prime} & B_{\mathrm{tcsc}}^{\prime}
\end{array}\right], \\
C_{\text {new }} & =\left[\begin{array}{ll}
C_{\text {sys } 1}^{\prime} & C_{1 \mathrm{tcsc}} \\
C_{\text {sys2 }}^{\prime} & C_{2 \mathrm{tcsc}}
\end{array}\right], \\
D_{\text {new }} & =\left[\begin{array}{ll}
D_{\text {sys11 }}^{\prime} & D_{\text {sys12 }}^{\prime} \\
D_{1 \mathrm{tcsc}}^{\prime} & D_{2 \mathrm{tcsc}}^{\prime}
\end{array}\right], \\
E_{\text {new }} & =\left[\begin{array}{ll}
E_{\text {sys-tcsc }}, 0,0,0
\end{array}\right]^{\mathrm{T}} .
\end{aligned}
$$

Therefore, (14) can be changed to another form as follows:

$$
\begin{aligned}
\Delta \dot{x}_{\text {new }} & =A_{\text {new }} \Delta x+B_{\text {new }} \Delta y+E_{\text {new }} \Delta u, \\
0 & =C_{\text {new }} \Delta x+D_{\text {new }} \Delta y .
\end{aligned}
$$

\section{The Proposed Approach}

3.1. Gramian-Based Controllability and Observability. The system often has two properties, controllability and observability, which play an important role regarding the determination of the optimal location of TCSC in the power system. From that, the input and output variables need to be used in order to observe and control the system. Therefore, (16) can be redescribed in a state-space form as follows:

$$
\begin{aligned}
& \dot{x}(t)=A x(t)+B u(t), \\
& y(t)=C x(t)+D u(t),
\end{aligned}
$$

where

$x(t)$ is the state vector,

$y(t)$ is the output vector,

$u(t)$ is the input vector,

$A$ is the state matrix,

$B$ is the control matrix,

$C$ is the output matrix,

$D$ is the feed-forward matrix.

\subsubsection{Controllability}

Definition 1. System (17) is controllable over the interval $\left[t_{0}, t_{1}\right]$, if any states $\left(x_{0}, x_{1} \in \mathscr{R}\right)$, there exists the input $u(t)$ : $\left[t_{0}, t_{1}\right] \rightarrow \mathscr{R}$, that drives the system from $x_{0}\left(t_{0}\right)$ to $x_{1}\left(t_{1}\right)$; this is true: the system is completely controllable despite the initial time and state.

Every actuator in the power systems is the energy that can be limited, such that controllability matrix has been used for the purpose of dealing with the amount of input energy. This input energy is required to reach a given state from the origin. The property of controllability of the system can be described in a quantitative manner by the controllability function. Typically, it is defined for dynamic system as the minimum input energy that necessary drives the system from state $x(-T)=0$ to state $x(0)=x_{0}$ and can be given as [46]

$$
\begin{aligned}
L_{c}\left(x_{0}\right)=\underset{u \in L_{2}(-T, 0)}{\operatorname{minimize}} & \int_{-T}^{0}\|u(t)\|^{2} d t, \\
\text { subject to: } & \dot{x}(t)=A x(t)+B u(t), \\
& x(-T)=0, \\
& x(0)=x_{0} .
\end{aligned}
$$

It can be proven that the above system (17) has the transient controllability function, which is given as follows:

$$
L_{c}\left(x_{0}\right)=\frac{1}{2} x_{0}^{\mathrm{T}} G_{c}^{-1}(T) x_{0} .
$$

Remark 2. For the real dynamic system, $L_{c}\left(x_{0}\right)$ is large and the state $x_{0}$ is difficult to reach. Thus, the system is uncontrollable.

Remark 3. Matrices $A$ and $B$ of the above system (17) are controllable in the time range $\left(t_{0}, t_{1}\right)$, if and only if the controllability matrix $\Delta$ defined as (20) has full rank $n$, where $n$ is the number of states. Notice that the controllability matrix has dimension $n \times n m$, where $m$ is the dimension of the input vector $u$.

$$
\Delta=\left[\begin{array}{lll}
B & A B & A^{2} B \cdots A^{n-1} B
\end{array}\right] .
$$

Remark 4. Matrices $A$ and $B$ of the above system (17) are controllable if and only if the controllability Gramian matrix 
$G_{c}(T)$ on horizon $\mathrm{T}$ defined as (21) has full rank $n$ and is positive definite.

$$
G_{c}(T)=\int_{0}^{T} \exp (A t) B B^{\mathrm{T}} \exp \left(A^{\mathrm{T}} t\right) d t .
$$

3.1.2. Observability. The system states are the internal variables, which are hard to directly measure but the outputs can be measured easily at the same time. The observability property plays an important role in the analysis of optimal location.

Definition 5. System (17) is observable over the interval $\left[t_{0}, t_{1}\right]$, if any states $x_{0}, x_{1} \in \mathscr{R}$, there exists the output $y(t)$ : $t_{0} \leq t \leq t_{1}<\infty$, assuming that the input $u(t)$ is known; this is true: the system is observable despite the initial time and state.

The observability property of the system can be characterized in a quantitative manner by the observability function. It is defined for dynamic system as output energy generated by the state $x(0)=x_{0}($ when $u=0)$ and is given as [46]

$$
\begin{array}{cl} 
& L_{o}\left(x_{0}\right)=\frac{1}{2} \int_{-T}^{0}\|y(t)\|^{2} d t, \\
\text { subject to: } & x(0)=x_{0}, \\
& u \equiv 0 .
\end{array}
$$

It can be proven that system (17) has the transient observability function, which is given as follows:

$$
L_{o}\left(x_{0}\right)=\frac{1}{2} x_{0}^{\mathrm{T}} G_{o}^{-1} x_{0} .
$$

Remark 6. For the real dynamic system, $L_{o}\left(x_{0}\right)$ measures the effect of the initial condition on the output; if $L_{o}\left(x_{0}\right)$ is small, the effect of $x_{0}$ in the output is confined, such that it is difficult to reach state $x_{0}$. Thus, the system is unobservable.

Remark 7. Matrices $A$ and $C$ of the above system (17) are observable in the interval $\left[t_{0}, t_{1}\right]$, if and only if the observability matrix $\nabla$ defined as (24) has full rank $n$, where $n$ is the number of states. Notice that the observability matrix has dimension $n p \times n$, where $p$ is the dimension of the output vector $y$.

$$
\nabla=\left[\begin{array}{c}
C \\
C A \\
C A^{2} \\
\vdots \\
C A^{n-1}
\end{array}\right] .
$$

Remark 8. Matrices $A$ and $C$ of the above system (17) are observable if and only if the observability Gramian matrix $G_{o}(T)$ on horizon T defined as (25) has full rank $n^{\prime}$ and is positive definite.

$$
G_{o}(T)=\int_{0}^{T} \exp \left(A^{\mathrm{T}} t\right) C^{\mathrm{T}} C \exp (A t) d t .
$$

If system (17) is asymptotically stability around the origin, the controllability and observability functions can be given by

$$
\begin{aligned}
& L_{c}=\underset{\substack{u \in L_{2}(-\infty, 0) \\
x(0)=x_{0} \\
x(-\infty)=0}}{\operatorname{minimize}} \frac{1}{2} \int_{-\infty}^{0}\|u(t)\|^{2} d t, \\
& L_{o}=\frac{1}{2} \int_{0}^{\infty}\|y(t)\|^{2} d t, \quad x(0)=x_{0}, u \equiv 0 .
\end{aligned}
$$

Formal remarks for controllability and observability are white and black. In reality, some states are very calamitous to control or have little effect on the outputs. The degree of observability and controllability can be evaluated by the sizes of $G_{c}$ and $G_{o}$ with infinite time horizon:

$$
\begin{aligned}
& G_{c}(0, \infty)=\lim _{T \rightarrow \infty} \int_{0}^{T} \exp (A t) B B^{\mathrm{T}} \exp \left(A^{\mathrm{T}} t\right) d t, \\
& G_{0}(0, \infty)=\lim _{T \rightarrow \infty} \int_{0}^{T} \exp \left(A^{\mathrm{T}} t\right) C^{\mathrm{T}} C \exp (A t) d t .
\end{aligned}
$$

Notice that $G_{c}$ and $G_{o}$ satisfy the following differential expression:

$$
\begin{aligned}
& \frac{d G_{c}(0, t)}{d t}=A G_{c}(0, t)+G_{c}(0, t) A^{\mathrm{T}}+B B^{\mathrm{T}}, \\
& \frac{d G_{o}(0, t)}{d t}=A^{\mathrm{T}} G_{o}(0, t)+G_{o}(0, t) A+C^{\mathrm{T}} C .
\end{aligned}
$$

It is hard to directly calculate the Gramian matrices from expression (27) because they consist of an exponential matrix and an integral. If all the eigenvalues of $A$ have strictly negative real parts, as $T \rightarrow \infty$, there exists an easier way to calculate these matrices by solving the Lyapunov expression (29) below [47], which can be solved using MATLAB via gram. The obtained solutions are the unique positive definite.

$$
\begin{aligned}
& A G_{c}+G_{c} A^{\mathrm{T}}+B B^{\mathrm{T}}=0, \\
& A^{T} G_{o}+G_{o} A+C^{\mathrm{T}} C=0 .
\end{aligned}
$$

Obviously, expression (29) shows that (i) the property of observability Gramian matrix depends on the output matrix $A$ and state matrix $C$; accordingly, the output energy can be affected by properly choosing this matrix. (ii) The property of controllability Gramian matrix depends on the control matrix $A$ and state matrix $B$; accordingly, the control energy can be affected by properly choosing this matrix. (iii) When the system is only detectable, the Gramian matrices will be only some nonnegative definite matrices.

In particular, we can pose the problem seeking the minimum input energy (i.e., the control energy) that must derive system (17) from the initial state $x_{0}$ to a final state $x_{t_{f}}$ at time $t=t_{f}$ and this energy is defined by [34]

$$
\begin{aligned}
J= & \left(\exp \left(A t_{f}\right) x_{0}-x_{t_{f}}\right)^{\mathrm{T}} \\
& \cdot\left(G_{c}-\exp \left(A t_{f}\right) G_{c} \exp \left(A^{\mathrm{T}} t_{f}\right)\right)^{\mathrm{T}} \\
& \cdot\left(\exp \left(A t_{f}\right) x_{0}-x_{t_{f}}\right)
\end{aligned}
$$


Clearly, expression (30) shows that minimizing $J$ is the same as maximizing the Gramian matrix $G_{c}$. Furthermore, maximizing the transmitted total energy (potential and kinetic energy) from the actuators to the structure for a given input energy, it can be obtained as follows

$$
E=\int_{0}^{\infty}\left(E_{p}(t)+E_{k}(t)\right) d t=\int_{0}^{\infty} x(t) x^{\mathrm{T}}(t) d t
$$

where $E_{k}$ is the kinetic energy and $E_{p}$ is the potential energy. In this paper, we suppose that the input is a Dirac impulse that excites all frequencies and $x(t)=\exp (A t) B$ is the impulse matrix. Hence, the obtained energy is the weighted trace of controllability Gramian $[34,48]$ :

$$
E=\operatorname{trace}\left(G_{c}\right)=\sum_{i=1}^{n} \sigma_{i}
$$

where $\sigma_{i}$ is the $i$ th Hankel singular value. The decomposition of this value denotes spatial energy decomposition contained in the impulse response. The sum of the singular values denotes the energy total in which each of the singular value represents the energy in a particular direction. The optimal location is determined based on the maximization of the total energy of the system at the fault clearing time. Therefore, (32) is a basis for selecting optimal control input.

3.2. Hankel-Norm Method. In order to compute easily, we focus on analyzing the infinite horizon Gramian. The Gramian matrices are computed by using expression (29) rather than solving expressions (21) and (25). However, it may be more difficult for analyzing the Vietnamese power system because that is a large-scale network. With such networks, the number of state variables could be big, leading to lost time for computing. In reality, determining the optimal location, we just need the number of major state variables that play an important role in order to analyze the linear system on the small-signal stability. Therefore, in order to solve this problem, the order reduction method is applied based on the computation of controllability and observability Gramian matrices introduced in [36].

3.2.1. Balanced Realization. Considering system (17), in order to make effect for this study, the so-called similarity transformation $M$ must need to modify the state variables and matrices of the system, but the output and input behaviors remain unchanged, such that the controllability and observability Gramian matrices satisfy the following:

$$
G_{c}=G_{o}=\left[\begin{array}{ccccc}
\sigma_{1} & 0 & 0 & \cdots & 0 \\
0 & \sigma_{2} & 0 & \cdots & 0 \\
\vdots & \vdots & \vdots & \ddots & \vdots \\
0 & 0 & 0 & \cdots & \sigma_{n}
\end{array}\right]
$$

$$
\text { with } \sigma_{1} \geq \sigma_{2} \geq \cdots \geq \sigma_{n} \geq 0 \text {. }
$$

The so-called similarity transformation transforms the controllability and observability Gramian matrices in the following way:

$$
\begin{aligned}
& \bar{G}_{c}=M G_{c} M^{\mathrm{T}}, \\
& \bar{G}_{o}=\left(M^{-1}\right)^{\mathrm{T}} G_{c} M^{-1} .
\end{aligned}
$$

However, the result turns out to be the invariant output and input behaviors, that is, $M G_{c} M^{\mathrm{T}}=\left(M^{-1}\right)^{\mathrm{T}} G_{c} M^{-1}$ (i.e., it relates to the Gramian of the original system by $M$ ).

Expression (34) constructs a state-space transformation that is considered with the form $f(t)=M x(t)$; substituting this state-space transformation into (17), the new system can be obtained:

$$
\begin{gathered}
\dot{f}(t)=\bar{A} f(t)+\bar{B} u(t), \\
y(t)=\bar{C} f(t)+\bar{D} u(t),
\end{gathered}
$$

where $\bar{A}=M A M^{-1} ; \bar{B}=M B ; \bar{C}=C M^{-1} ; \bar{D}=D$. Therefore, system (35) is a balanced form according to the given nonsingular transformation matrix $M$ to change the system in such a way that the controllability and observability Gramian matrices satisfy the following condition:

$$
\begin{aligned}
& \bar{G}_{c}=\bar{G}_{o}=\operatorname{diag}\left\{\sigma_{1}, \sigma_{1}, \ldots, \sigma_{n}\right\}, \\
& \sigma_{1} \geq \sigma_{1} \geq \cdots \geq \sigma_{n},
\end{aligned}
$$

in which $\sigma_{1}, \sigma_{2}, \ldots, \sigma_{n}$ are real and positive numbers called the Hankel singular values and the transformation matrix is determined by using the algorithm [47] as shown in the Appendix.

3.2.2. Order Reduction Realization. After obtaining the new system in a balanced form as shown in (35), the order reduction can be performed. The first step is to choose the order of $k$ based on the purposes of reduction. The author in [36] has considered general situation that the Hankel singular values satisfy the condition, that is,

$$
\begin{aligned}
\sigma_{1} & \geq \sigma_{2} \geq \cdots \geq \sigma_{k} \geq \sigma_{k+1}=\sigma_{k+2}=\cdots=\sigma_{k+r} \\
& >\sigma_{k+r+1} \geq \cdots \geq \sigma_{n}>0 .
\end{aligned}
$$

In this study, the system reduction is performed by eliminating all state variables corresponding to the Hankel singular values that are smaller than $10^{-3}$. Therefore, the system can be converted to the following order reduction form:

$$
\begin{gathered}
\dot{f}_{1}(t)=\bar{A}_{11} f_{1}(t)+\bar{A}_{12} f_{2}(t)+\bar{B}_{1} u(t) \\
\dot{f}_{2}(t)=\bar{A}_{21} f_{1}(t)+\bar{A}_{22} f_{2}(t)+\bar{B}_{2} u(t) ; \\
y(t)=\bar{C}_{1} f_{1}(t)+\bar{C}_{2} f_{2}(t)+\bar{D} u(t),
\end{gathered}
$$

$$
\bar{G}_{c}=\bar{G}_{o}=\bar{\Sigma},
$$

where $\bar{\Sigma}=\left[\left(\operatorname{diag}\left\{\sigma_{1}, \ldots, \sigma_{k}, \sigma_{k+r+1}, \ldots, \sigma_{n},\right\}\right)^{2}-\sigma_{k+1}^{2} \Psi_{r}\right]=$ $\left(\bar{\Sigma}_{1}^{2}-\sigma_{k+1}^{2} \Psi_{r}\right)$ is the nonsingular and diagonal matrix. The 
vectors $f_{1}(t)$ and $f_{2}(t)$ are obtained by decomposing the state variable $f(t)$ (i.e., $f(t)=\left[f_{1}(t) f_{2}(t)\right]^{\mathrm{T}}$ ) and have dimensions $n-r$ and $r$, respectively. $\Psi_{r}$ is the identity matrix and has dimension $r$. The matrices $\left(\bar{A}_{11}, \bar{A}_{12} ; \bar{A}_{21}, \bar{A}_{22}\right)^{2 \times 2},\left(\bar{B}_{1} ; \bar{B}_{2}\right)^{1 \times 2}$, and $\left(\bar{C}_{1}, \bar{C}_{2}\right)^{2 \times 1}$ are, respectively, partitioned from matrices $\bar{A}, \bar{B}$, and $\bar{C}$ in balanced system (35) corresponding to the partitioned Gramian.

If the number of inputs is more than that of outputs (the so-called all-pass dilation of the system), then, $\bar{A}, \bar{B}, \bar{C}$, and $\bar{D}$ are replaced by $\bar{A}^{\mathrm{T}}, \bar{B}^{\mathrm{T}}, \bar{C}^{\mathrm{T}}$, and $\bar{D}^{\mathrm{T}}$, respectively [49]. Thus, the components for the so-called all-pass dilation of the system are defined as follows:

$$
\begin{array}{rlr}
\widetilde{A} & =\bar{\Sigma}^{-1}\left(\sigma_{k+1}^{2} \bar{A}_{11}^{\mathrm{T}}+\bar{\Sigma}_{1} \bar{A}_{11} \bar{\Sigma}_{1}-\sigma_{k+1} \bar{C}_{1}^{\mathrm{T}} U \bar{B}_{1}^{\mathrm{T}}\right), \\
\widetilde{B} & =\bar{\Sigma}^{-1}\left(\bar{\Sigma}_{1} \bar{B}_{1}+\sigma_{k+1} \bar{C}_{1}^{\mathrm{T}} U\right), \\
\widetilde{C} & =\bar{C}_{1} \bar{\Sigma}_{1}+\sigma_{k+1} U \bar{B}_{1}^{\mathrm{T}}, \\
\widetilde{D} & =\bar{D}-\sigma_{k+1} U ; \\
& \bar{\Sigma}=\bar{\Sigma}_{1}^{2}-\sigma_{k+1}^{2} \Psi_{r},
\end{array}
$$

where $U$ is a unitary matrix and is solution of the following expression:

$$
\bar{B}_{2}+\bar{C}_{2}^{\mathrm{T}} U=0
$$

According to the conclusion in [49], the all-pass dilation of the system might be unstable. The final step is to compute the stable part of the all-pass dilation of the systems $\widetilde{A}^{\prime}$, $\widetilde{B}^{\prime}, \widetilde{C}^{\prime}$, and $\widetilde{D}^{\prime}$ based on the Hankel-norm method. As we consider a system that the number of inputs is more than the number of outputs, matrices $\widetilde{A}^{\prime}, \widetilde{B}^{\prime}, \widetilde{C}^{\prime}$, and $\widetilde{D}^{\prime}$ can be replaced by $\widetilde{A}^{\prime \mathrm{T}}, \widetilde{B}^{\prime \mathrm{T}}, \widetilde{C}^{\prime \mathrm{T}}$, and $\widetilde{D}^{\prime \mathrm{T}}$ based on the following error of approximation:

$$
\left\|T(s)-T_{k}(s)\right\|_{\infty}=\sigma_{k+1},
$$

where $T(s)$ and $T_{k}(s)$ are the transfer functions of the original and the $k_{\text {th }}$-order reduced systems.

Clearly, expression (41) shows that the largest error for all frequencies is equal to the Hankel singular value $k+1$. It allows us to select a suitable value of $k$. Therefore, the matrices of the reduced system are obtained as follows:

$$
\begin{aligned}
& A_{k}=\widetilde{A}^{\prime}, \\
& B_{k}=\widetilde{B}^{\prime}, \\
& C_{k}=\widetilde{C}^{\prime}, \\
& D_{k}=\widetilde{D}^{\prime} .
\end{aligned}
$$

3.3. Algorithm. The main objective is to dampen the active power and generator angle oscillations in the power system when the active power perturbation occurs in the transmission line. The proposed algorithm is used to determine the optimal location of TCSC based on the Gramian critical energy, and the flowchart of the algorithm is shown in Figure 5. The principles and details have been presented in Sections 3.1 and 3.2 and below is the interpretation.

First. Start.

Step 1. Place the TCSC controller in the line $i$ th $(i=1,2$, $3, \ldots, n)$.

Step 2. Run steady state.

Step 3. Generate the active power perturbation signal in the transmission line $j$ th $(j=1,2,3, \ldots, m)$ in which $m$ is large enough. For each contingency of the active power perturbation $j$, the observed output $v_{j}$ and control input $\xi_{j}$ were analyzed.

Step 4. Compute the matrices $A, B$, and $C$ corresponding to the placement $i$ and the active power perturbation signal $j$.

Step 5. Perform the order reduction for system based on the Hankel-norm method.

Step 6. Estimate the stable condition based on the state matrix $\left(A_{k}\right)_{i j}$ to exclude the unstable cases. If the condition is satisfied, Step 6 will be performed, and, vice versa, Step 1 will be iterated.

Step 7. Compute the controllability Gramian matrix of the new system (after performing the order reduction) corresponding to the active power perturbation signal $j$ th.

Step 8. Iterate the steps from 2 to 7 for computing a set of the active power perturbations $(j=1,2,3, \ldots, m)$.

Step 9. Compute the energy for each placement corresponding to a set of the active power perturbations $(j=$ $1,2,3, \ldots, m)$.

Step 10. Iterate the steps from 1 to 7 to calculate all the locations $(i=1,2,3, \ldots, n)$.

Step 11. Compare the maximum total energy to evaluate the optimal placement for TCSC controller.

Finally. End.

\section{Case Study}

The Vietnamese $500 / 220 \mathrm{kV}$ transmission system is used in the study to illustrate the effectiveness of the proposed method. This system consists of 29 substations of $500 \mathrm{kV}, 162$ substations of $220 \mathrm{kV}, 16$ double-circuit lines and 20 singlecircuit lines of $500 \mathrm{kV}, 205$ double-circuit lines and 67 singlecircuit lines of $220 \mathrm{kV}$, and 179 generator units. The generated total power is about $42,179 \mathrm{MW}$; the peak load demand is 


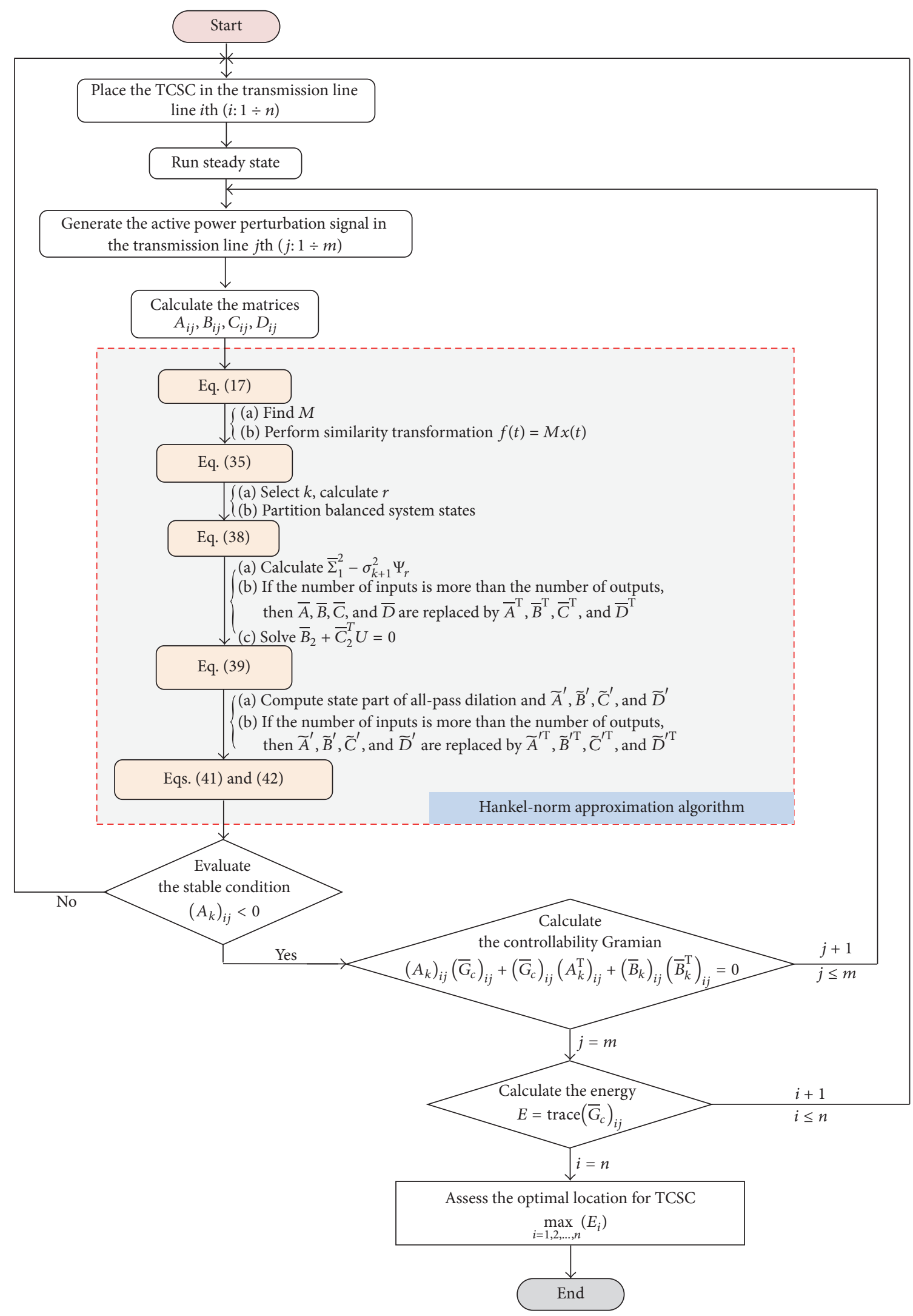

FIGURE 5: Flowchart of the proposed algorithm. 
TABLE 1: The existing compensation ratio on the $500 \mathrm{kV}$ system.

\begin{tabular}{|c|c|c|c|}
\hline $\begin{array}{l}\text { Case } \\
\text { number }\end{array}$ & $\begin{array}{l}\text { The line between } \\
\text { buses }\end{array}$ & $\begin{array}{l}\text { Compensation } \\
\text { ratio }(\%) \\
\end{array}$ & On circuit \\
\hline (1) & $\begin{array}{l}\text { NhoQuan-Hatinh } \\
\text { (NQ-HT) }\end{array}$ & 57.7 & 1 \\
\hline (2) & NQ-HT & 54.4 & 2 \\
\hline (3) & $\begin{array}{l}\text { VungAng-Danang } \\
\text { (VA-DN) }\end{array}$ & 64 & 1 \\
\hline (4) & VA-DN & 64 & 2 \\
\hline (5) & $\begin{array}{l}\text { Danang-Docsoi } \\
\text { (DN-DS) }\end{array}$ & 63 & - \\
\hline (6) & $\begin{array}{l}\text { Docsoi-Pleiku } \\
\text { (DS-Plei) }\end{array}$ & 58 & - \\
\hline (7) & $\begin{array}{l}\text { Thanhmy-Pleiku } \\
\text { (TM-Plei) }\end{array}$ & 52 & - \\
\hline (8) & $\begin{array}{l}\text { Pleiku-Daknong } \\
\text { (Plei-DakN) }\end{array}$ & 76 & - \\
\hline (9) & $\begin{array}{l}\text { Daknong-Caubong } \\
\text { (DakN-CB) }\end{array}$ & 61 & - \\
\hline (10) & $\begin{array}{l}\text { Pleiku-Dilinh } \\
\text { (Plei-DL) }\end{array}$ & 70 & - \\
\hline (11) & $\begin{array}{l}\text { Dilinh-Tandinh } \\
\text { (DL-TD) }\end{array}$ & 59 & - \\
\hline (12) & $\begin{array}{l}\text { Pleiku-Caubong } \\
\text { (Plei-CB) }\end{array}$ & 70 & - \\
\hline
\end{tabular}

about 40,703 MW. The power system simulation engineering (PSS/E) program is used to analyze the transient and smallsignal stability and MATLAB is used to calculate Gramian matrices and perform order reduction. All dynamic models such as generators, excitation systems, transmission lines, and loads are modeled by using PSS/E (from dynamic model library) [50], and all dynamic parameters are taken from [2] and the Vietnamese national load dispatch center (NLDC). The single-line diagram and the result of load flow calculation of the $500 \mathrm{kV}$ voltage level are given in Figure 2.

The optimal placement of TCSC in the Vietnamese network is determined based on (i) a combination of the controllability Gramian critical energy with the order reduction method on the small-signal stability analysis applied to search for several feasible locations; (ii) the transient stability analysis performed to compare and determine optimal locations through various simulation cases.

In addition, the existing series compensation ratio on the Vietnamese $500 \mathrm{kV}$ power system is considered to calculate and listed out in Table 1.

4.1. Determine Feasible Locations. The correctness of the proposed method for the optimal location of TCSC is verified on the small-signal analysis; the dynamic model of TCSC is chosen as shown in Figure 4 [34]. We suppose the compensation capacity of TCSC is set to the range $[0.75,1.2]$ of the uncompensated line. The active power perturbation signal in the line is considered as the input signal for the TCSC controller [51]. The optimal method in [38] was used to determine the parameters for the TCSC controller, and these parameters are listed in Table 2 [34].
TABLE 2: Parameters of the TCSC controller.

\begin{tabular}{lccc}
\hline Parameter & Value & Parameter & Value \\
\hline$T_{1}$ & $0.1 \mathrm{sec}$ & $K$ & 0.75 \\
$T_{2}$ & $0.1 \mathrm{sec}$ & $X_{\max }$ & $1.2 X_{\text {line }}$ p.u. \\
$T_{3}$ & $0.4 \mathrm{sec}$ & $X_{\min }$ & $0.25 X_{\text {line }}$ p.u. \\
$T_{w}$ & $10 \mathrm{sec}$ & $T_{\text {tcsc }}$ & $0.015 \mathrm{sec}$ \\
\hline
\end{tabular}

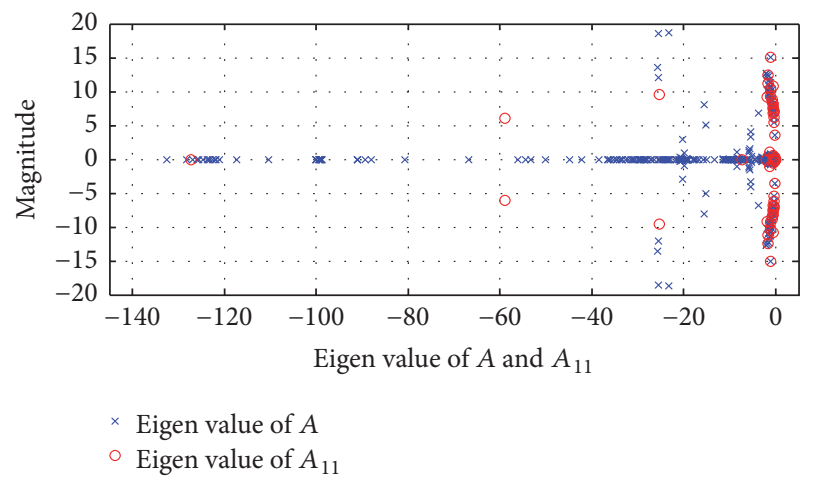

FIGURE 6: Eigenvalue distribution before and after using balanced reduction.

The studied contingency cases of active power perturbation were selected from single-line outage cases on the basis of the real power flow performance index (PI) introduced in [36], as follows:

$$
\mathrm{PI}=\sum_{i=1}^{n l} \frac{v_{i}}{2 \vartheta}\left(\frac{P_{l i}}{P_{l i}^{\max }}\right)^{2 \vartheta},
$$

where $P_{l i}$ and $P_{l i}^{\max }$ are the real power and rated power flow of the line $i$, respectively, $\vartheta$ is the exponent, and $v_{i}$ is a the real nonnegative weighting factor for line $i$. In this study, the value of the exponent was selected as 2.0 and 1.0 is the value of $v_{i}$.

Observing from (43) that all the power flows in the lines are within their limits, PI is small and, vice versa, it reaches out a high value when there are overloads. The studied contingency cases are listed in Table 3 by using (43); this corresponds to the active power perturbation signal in the line of the Vietnamese network.

The Vietnamese $500 / 220 \mathrm{kV}$ power system created the state matrix $(A)$ having dimension $[2371 \times 2371]$ that corresponds with 179 generator units. By performing the equivalence of 179 generator units into 45 equivalent generator units, the state matrix now has dimension [515 $\times 515]$. A TCSC controller has three states as the transducer, washout, and lead/lag added in the system. Therefore, the state matrix dimension of the equivalent power system model is [518 $\times$ 518].

Once again, the state matrix of the system is reduced the dimension by applying the balanced order reduction method to eliminate the singular values that are smaller than $10^{-3}$. Figure 6 shows the eigenvalue of the system state matrix before and after using balanced reduction. Therefore, the state matrix $\left(A_{11}\right)$ of the new system has the dimension [45 $\left.\times 45\right]$. 
TABLE 3: Studied contingency cases.

\begin{tabular}{|c|c|}
\hline Case number & $\begin{array}{l}\text { Active power perturbation signal in the line } \\
\text { between buses }\end{array}$ \\
\hline (1) & NhoQuan-HoaBinh (NQ-HB) \\
\hline (2) & VietTri-Pitoong (VT-PT) \\
\hline (3) & ThangLong-PhoNoi (TL-PN) \\
\hline (4) & NhoQuan-SonLa (NQ-SL) \\
\hline (5) & Pitoong-HoaBinh (PT-HB) \\
\hline (6) & QuangNinh-HiepHoa (QN-HH) \\
\hline (7) & HiepHoa-VietTri (HH-VT) \\
\hline (8) & HiepHoa-Pitoong (HH-PT) \\
\hline (9) & Pitoong-SonLa (PT-SL) \\
\hline$(10)$ & QuangNinh-ThangLong (QN-TL) \\
\hline$(11)$ & QuangNinh-PhoNoi (QN-PN) \\
\hline$(12)$ & QuangNinh-MongDuong (QN-MD) \\
\hline (13) & ThuongTin-Nho Quan (TT-NQ) \\
\hline$(14)$ & ThuongTin-PhoNoi (TT-PN) \\
\hline (15) & VungAng-HaTinh (VA-HT) \\
\hline (16) & DaNang-ThanhMy (DN-TM) \\
\hline$(17)$ & SongMay-TanUyen (SM-TY) \\
\hline (18) & SongMay-TanDinh (SM-TD) \\
\hline (19) & NhaBe-PhuLam (NB-PL) \\
\hline (20) & TanDinh-CauBong (TD-CB) \\
\hline$(21)$ & PhuLam-MyTho (PL-MT) \\
\hline$(22)$ & PhuLam-DucHoa (PL-DH) \\
\hline (23) & DucHoa-MyTho (DH-MT) \\
\hline$(24)$ & MyTho-DuyenHai (MT-DH) \\
\hline (25) & OMon-MyTho (OM-MT) \\
\hline$(26)$ & VinhTan-SongMay (VT-SM) \\
\hline$(27)$ & PhuMy-NhaBe (PM-NB) \\
\hline$(28)$ & CauBong-PhuLam (CB-PL) \\
\hline (29) & NhaBe-MyTho (NB-MT) \\
\hline$(30)$ & CauBong-DucHoa (CB-DH) \\
\hline$(31)$ & SongMay-PhuMy (SM-PM) \\
\hline
\end{tabular}

Figure 7 shows the frequency response of the system before and after using balanced reduction. Obviously, the input-output behaviors of the system in both cases at the bandwidth range from $10^{-\infty}$ to $10^{5}$ are the same with the same control signal. The bandwidth has a range from $10^{5}$ to $10^{\infty}$; the frequency response of the reduced system is flat (a straight line) since its order is smaller than the original system. Therefore, we can conclude that two systems are equivalent in terms of the input-output behaviors.

Figure 8 shows the Hankel singular values after performing the balanced realization reduction technique. It can be observed from this figure that the 45 Hankel singular values have been finally retained in comparison with the 2374 state variables of the original system (including state variables of TCSC controller). This acquired singular values are smaller than the chosen threshold $10^{-3}$ (choice of this study). The

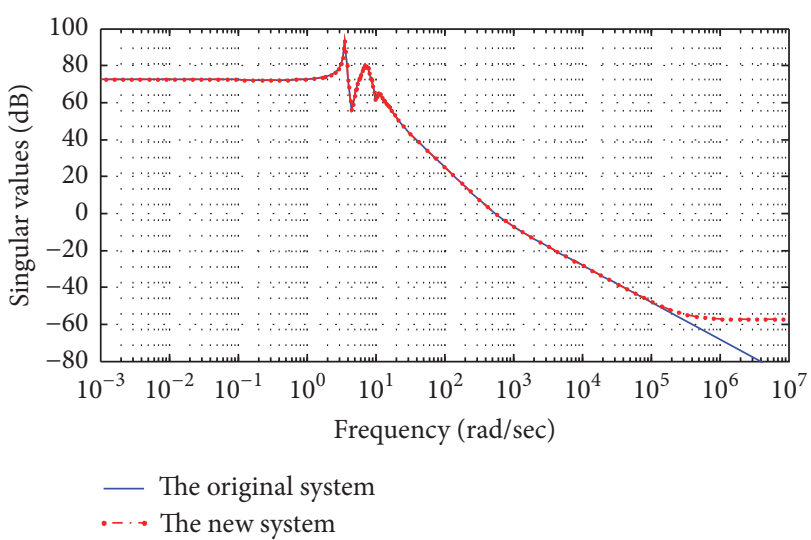

FIGURE 7: Frequency response of the original and reduced system.

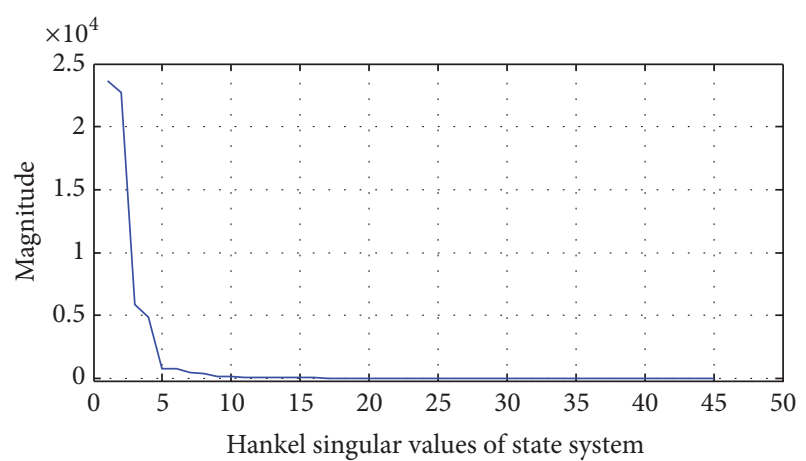

FIGURE 8: Distribution of Hankel singular values.

eigenvalues distribution of the system before and after using balanced reduction is plotted in Figure 8. Therefore, the reduced system has 45 state variables, which can fully meet effects of the network to calculate the Gramian matrices.

The controllability Gramian energy indices were determined based on the proposed algorithm as shown in Section 3.3 and the active power perturbation signals in the line as listed in Table 3 when the transient horizon is equal to infinity $(T=\infty)$ are given in Table 4 , in which we only give several feasible locations having the high total energy value. From this table, it can be seen that the line PLei-CB is the suitable location for TCSC controller because it has the maximum total energy value; it means that the energy needed to drive the controllable state variables is smaller than other cases. Therefore, the line between buses PLei and CB is considered as the best location to install the TCSC controller.

4.2. Retest Transient Stability. To verify the effectiveness of the proposed method, several dynamic cases are analyzed based on the transient stability to compare the suitable location (the line between buses PLei and CB) and other feasible ones (as shown in Table 4). The optimal location of the TCSC controller is determined based on the rotor angle oscillation damping of the generator units having the large output power and the active power damping of the transmission line. In addition, the power distribution on 
TABLE 4: Energy values according to several feasible locations of TCSC.

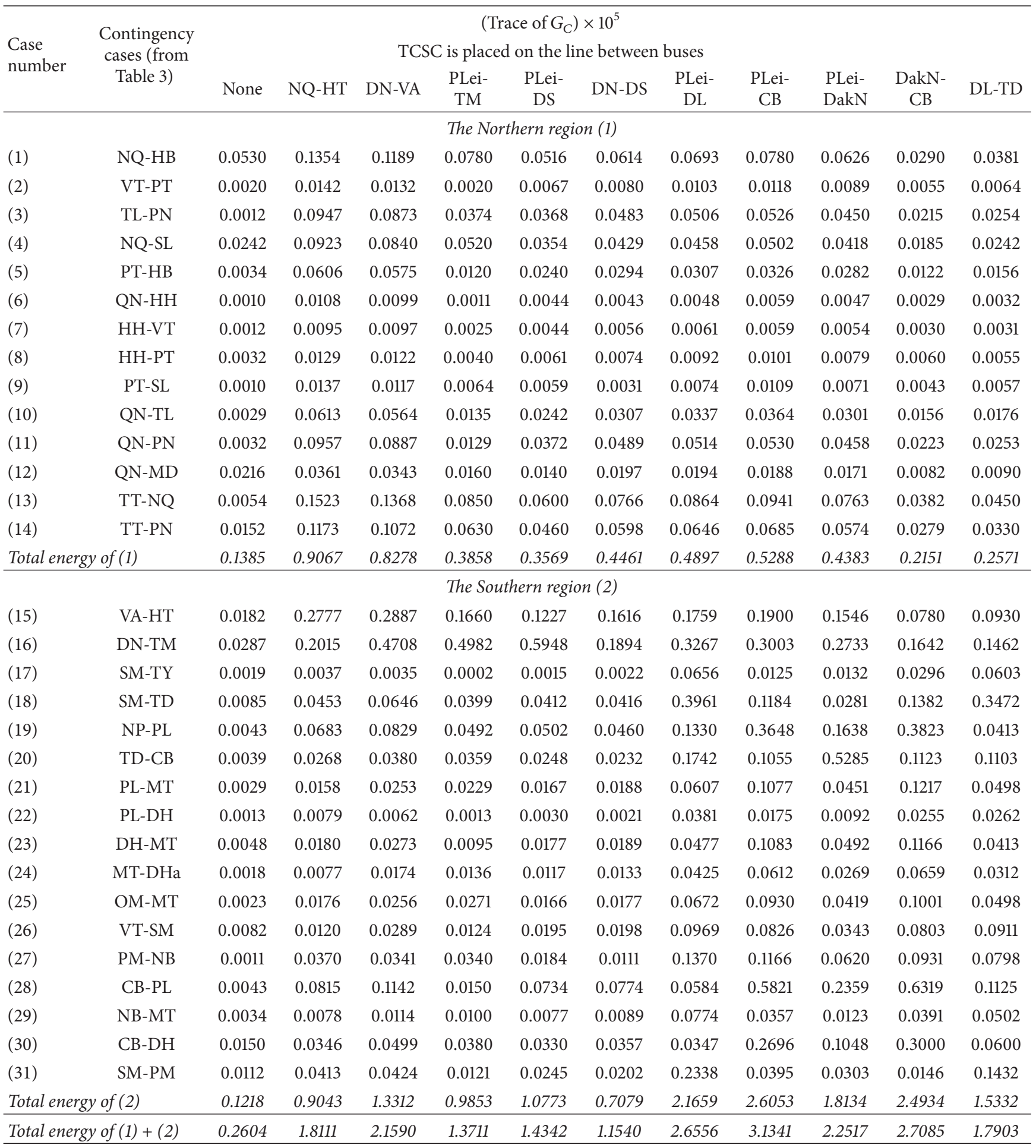

$500 / 220 \mathrm{kV}$ system at maximum and/or minimum load is considered. For maximum load, the transferred power on the $500 \mathrm{kV}$ system from the North and Central to the South is very high. Thus, when the three-phase fault occurs on the lines such as Vungang (VA)-Danang (DN), and PLeiku (PLei)-DiLinh (DL), the system lost serious stability. The three-phase fault scenarios are considered in this study as listed in Table 5 and can be summarized briefly as follows.

Scenario Number 1. A solid three-phase fault occurs at $1 \mathrm{sec}$ on the line $500 \mathrm{kV} \mathrm{DN-VA}$ of circuit 1, close to the bus $500 \mathrm{kV}$ $\mathrm{DN}$, and is cleared after $0.15 \mathrm{sec}$ by tripping the faulted line. 


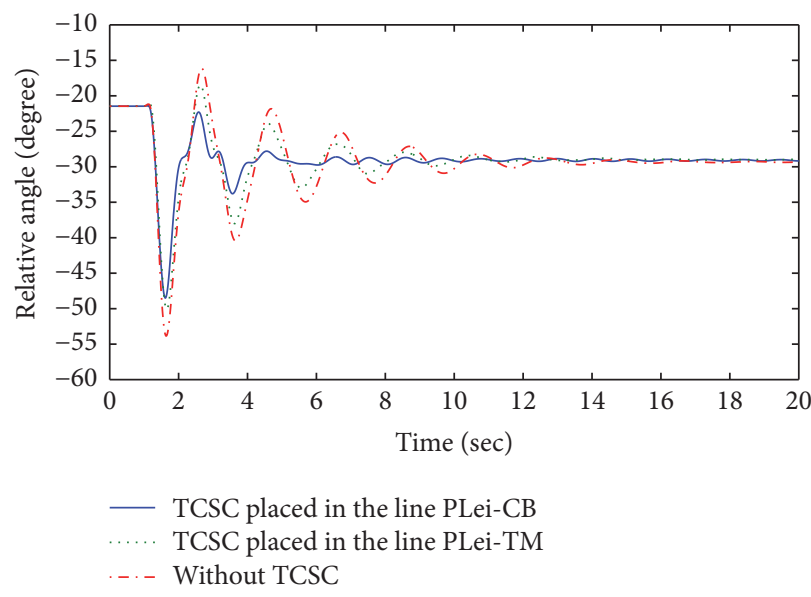

(a)

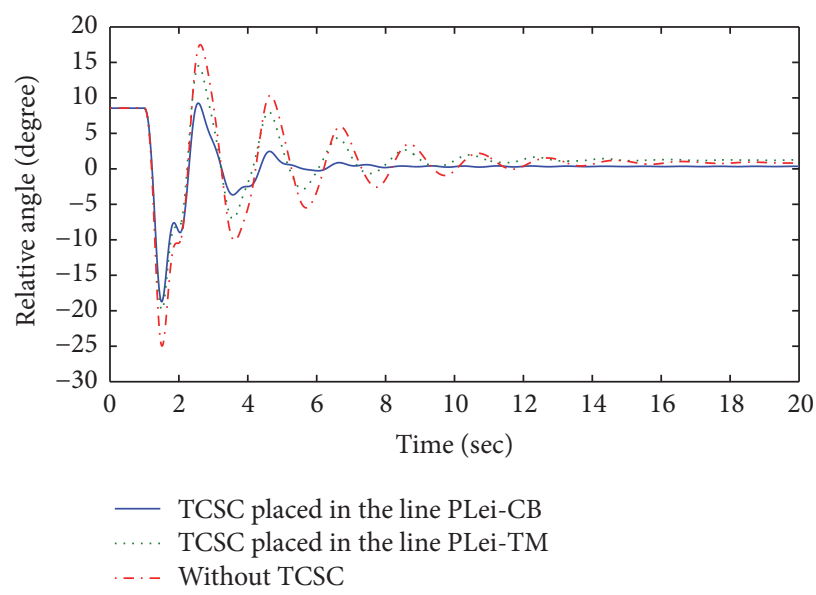

(c)

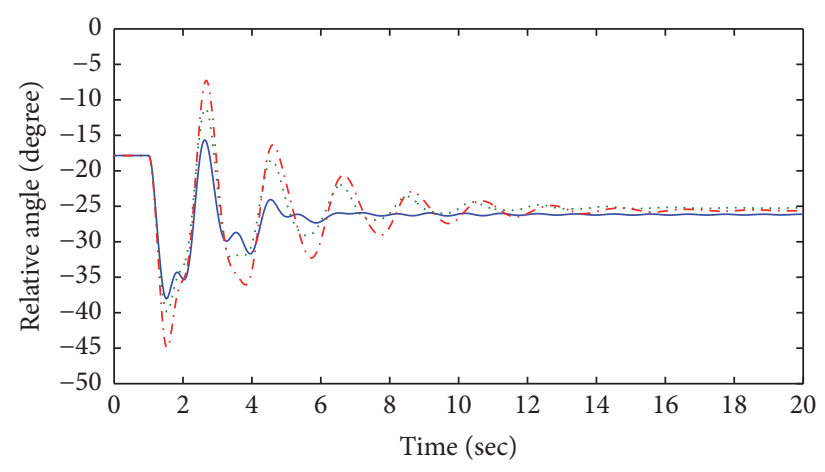

- TCSC placed in the line PLei-CB

TCSC placed in the line PLei-TM ...- Without TCSC

(b)

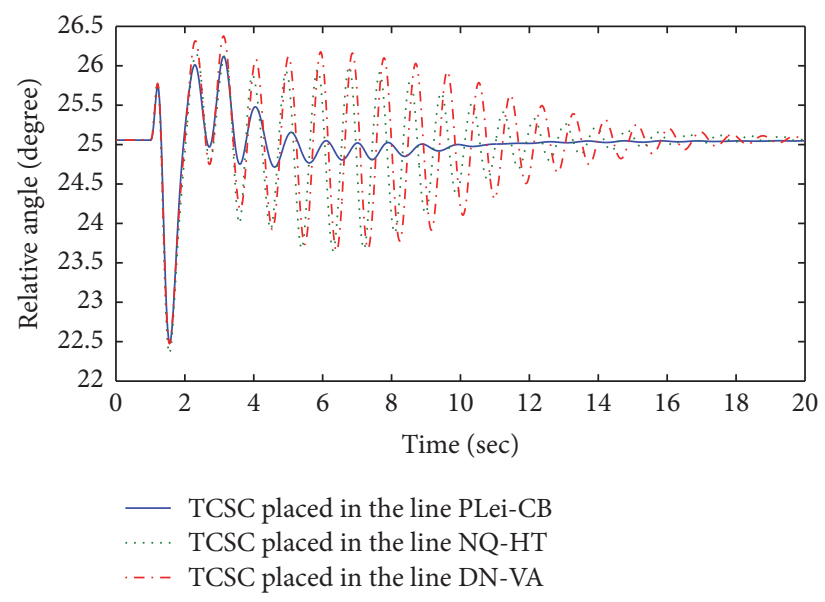

(d)

Figure 9: The relative angle oscillations of generators: (a) Yaly; (b) PhuMy-3; (c) VinhTan; (d) QuangNinh.

TABLE 5: Scenarios on three-phase fault for test of transient stability.

\begin{tabular}{lcc}
\hline Case number & Fault is nearly bus & Line outage \\
\hline$(1)$ & $500 \mathrm{kV} \mathrm{DN}$ & $500 \mathrm{kV} \mathrm{DN}-\mathrm{VA}$ \\
$(2)$ & $500 \mathrm{kV}$ Plei & $500 \mathrm{kV}$ PLei-DL \\
$(3)$ & $500 \mathrm{kV} \mathrm{CB}$ & $500 \mathrm{kV}$ TD-CB \\
$(4)$ & $500 \mathrm{kV} \mathrm{NQ}$ & $500 \mathrm{kV}$ NQ-TT \\
\hline
\end{tabular}

Scenario Number 2. A solid three-phase fault occurs at $1 \mathrm{sec}$ on the line $500 \mathrm{kV}$ PLei-DL, close to the bus $500 \mathrm{kV}$ Plei, and is cleared after $0.15 \mathrm{sec}$ by tripping the faulted line.

Scenario Number 3. A solid three-phase fault occurs at $1 \mathrm{sec}$ on the line $500 \mathrm{kV}$ TD-CB, close to the bus $500 \mathrm{kV}$ TD, and is cleared after $0.15 \mathrm{sec}$ by tripping the faulted line.

Scenario Number 4. A solid three-phase fault occurs at $1 \mathrm{sec}$ on the line $500 \mathrm{kV}$ TT-NQ, close to the bus $500 \mathrm{kV} \mathrm{NQ}$, and is cleared after $0.15 \mathrm{sec}$ by tripping the faulted line.
Case 1. The simulation was done on scenario number 1 based on the relative angle oscillations of generator, supposing that the system has been operating at maximum load in order to compare the difference locations of TCSC (that are on the lines PLei-CB and PLei-TM) and without TCSC. Figure 9 plots the relative angle oscillations of generators of Yaly, PhuMy-3, VinhTan, and QuangNinh. Figures 9(a)9(c) show the TCSC placed in the line PLei-CB, the angle oscillations of generators Yaly, PhuMy-3, and VinhTan are damped out in about 6 sec compared to that in the line PLeiTM and without TCSC controller. In particular, it can be observed from Figure 9(d) that the oscillations of generator QuangNinh are damped out faster than the case when the TCSC is placed in the line PLei-CB.

Case 2. The simulation of this case was done on scenario number 2 based on the active power of the line and the relative angle oscillations of generators to compare the suitable location of TCSC and other feasible locations as NQ-HT and DN-VA. In this case, the system has been operating at maximum load. Observing from Figure 10, TCSC is placed in the line PLei-CB; the transient response is significantly 


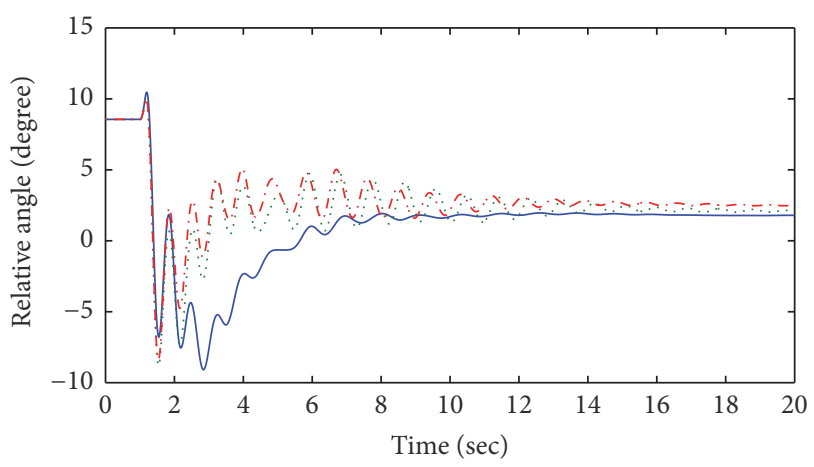

TCSC placed in the line PLei-CB
$\ldots .$. TCSC placed in the line NQ-HT
..$\cdot$ TCSC placed in the line DN-VA

(a)

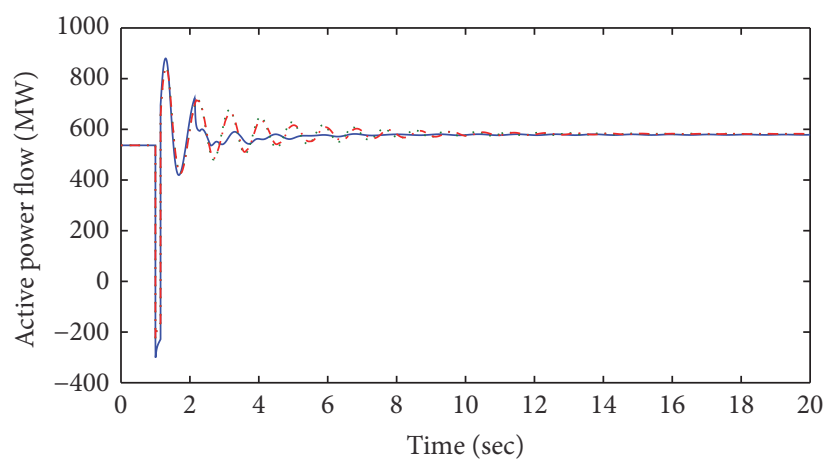

_ TCSC placed in the line PLei-CB TCSC placed in the line NQ-HT -. - TCSC placed in the line DN-VA

(b)

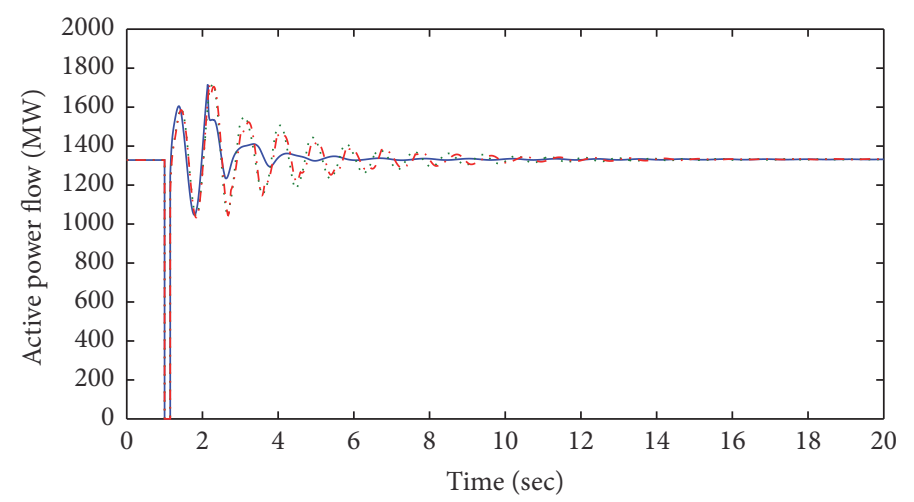

_ TCSC placed in the line PLei-CB

TCSC placed in the line NQ-HT

-. - TCSC placed in the line DN-VA

(c)

FIGURE 10: The transient response: (a) the relative angle oscillations of generator VinhTan; (b) the active power oscillations in the line CauBong-DucHoa; (c) the active power oscillations in the line Pleilu-ThanhMy.

improved compared with that in the two another locations. This could easily be construed from Table 4: the total energy value when TCSC is placed in the line PLei-CB is the larger.

Case 3. From Table 4, it can be seen that the other feasible locations (DakN-CB and PLei-DL) have the second and third highest total energy values, respectively. The third simulation was carried out for scenario number 3 to compare these feasible locations with the suitable location (PLei-CB). In this case, the system is considered as operating at maximum load. Figure 11 plots the system response as the relative angle oscillations of generators (VinhTan and DuyenHai) and the active power oscillations in the lines (HaTinh-VungAng and PLeilu-ThanhMy). It can be observed from Figures 11(a)11(d) that with TCSC placed in the line between PLei and $\mathrm{CB}$ the oscillations are damped faster compared with two other instances. Evidently, the effect is obtained in terms of oscillation damping from other two feasible locations to be the same because they have the same value of total energy value. Figure 11(e) shows the response of TCSC controller; it can be observed in this figure that the TCSC has injected the reactance to the grid during the period of fault and that the best influence is obtained in terms of reactive power flow output of TCSC when it is placed in the line PLei-CB.

Case 4. Observing from Table 4, the highest total energy value was obtained on the electric power supply system in the Northern region corresponding to the TCSC controller placed in the line NQ-HT. Contrarily, in this controller placed in the line Plei-CB, the highest total energy value was obtained on the electric power supply system in the Southern region. This simulation case was done on scenario number 4 to compare two locations having the highest total energy value at two regions. In this case, we suppose that the system has been operating at maximum load. Figure 12 plots the relative angle oscillations of generators QuangNinh and VinhTan. Evidently, it can be observed from this figure that TCSC is placed in line PLei-CB; oscillations are damped faster compared to placement in the line NQ-HT despite the far 


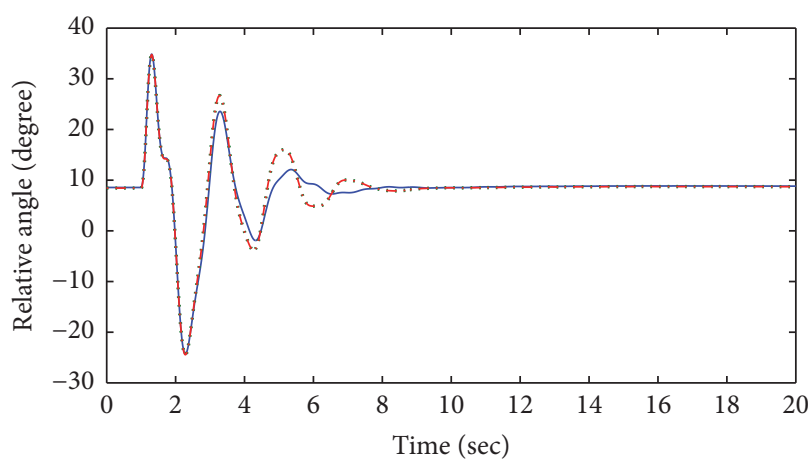

- TCSC placed in the line PLei-CB

..... TCSC placed in the line Plei-DL

... TCSC placed in the line DakN-CB

(a)

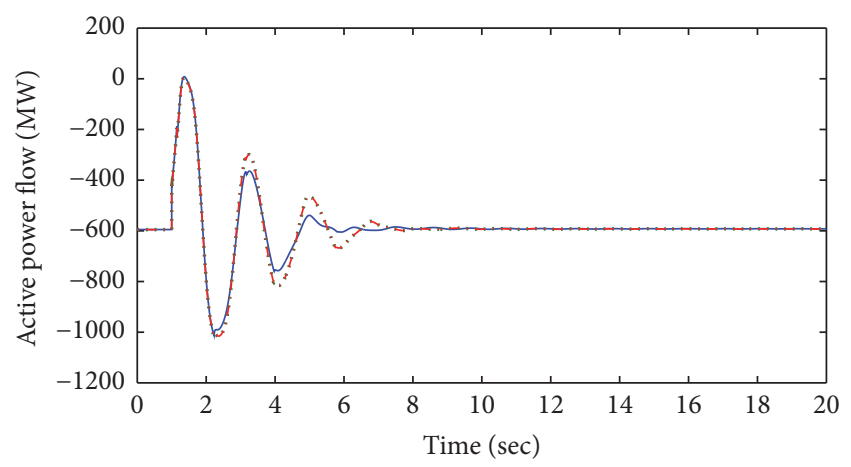

- TCSC placed in the line PLei-CB

..... TCSC placed in the line Plei-DL

... TCSC placed in the line DakN-CB

(c)

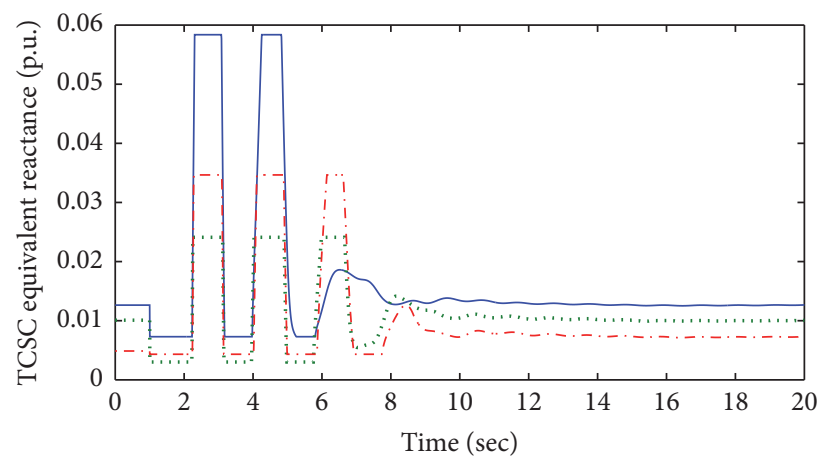

- TCSC placed in the line PLei-CB

...... TCSC placed in the line Plei-DL

... TCSC placed in the line DakN-CB

(e)

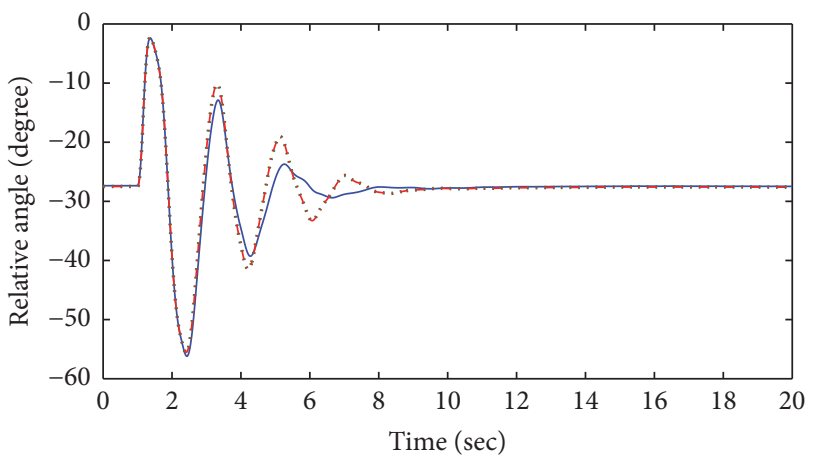

- TCSC placed in the line PLei-CB

..... TCSC placed in the line Plei-DL

... TCSC placed in the line DakN-CB

(b)

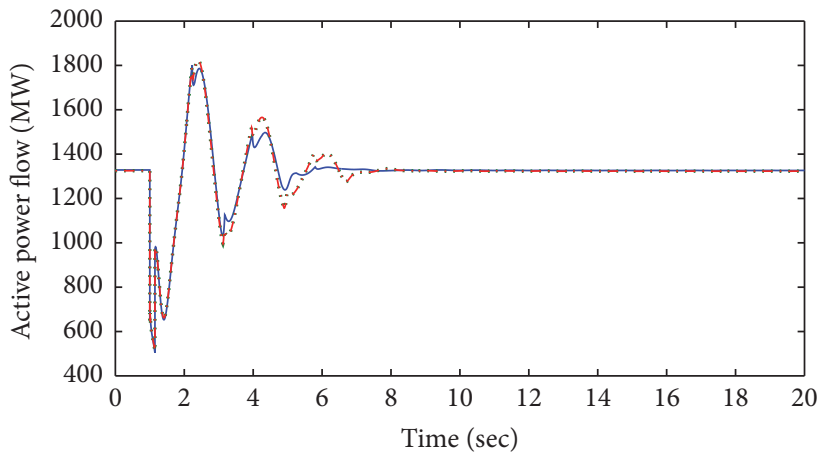

- TCSC placed in the line PLei-CB

...... TCSC placed in the line Plei-DL

...- TCSC placed in the line DakN-CB

(d) 


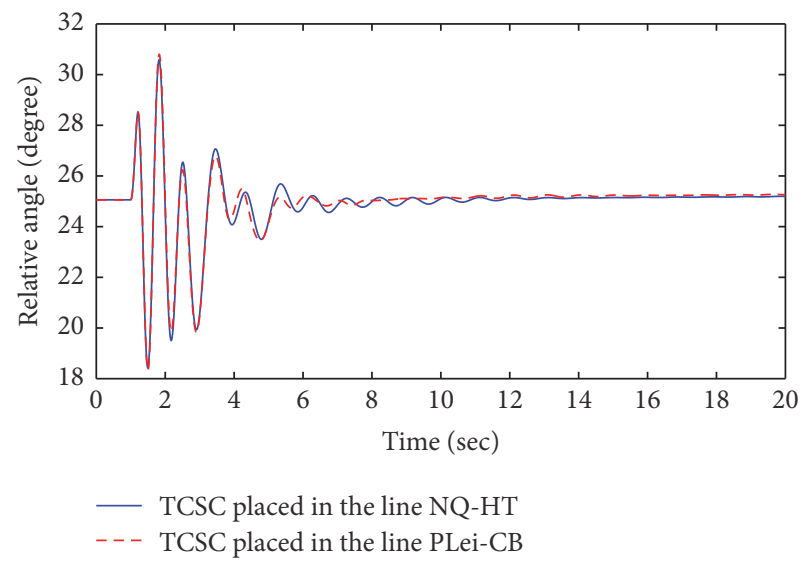

(a)

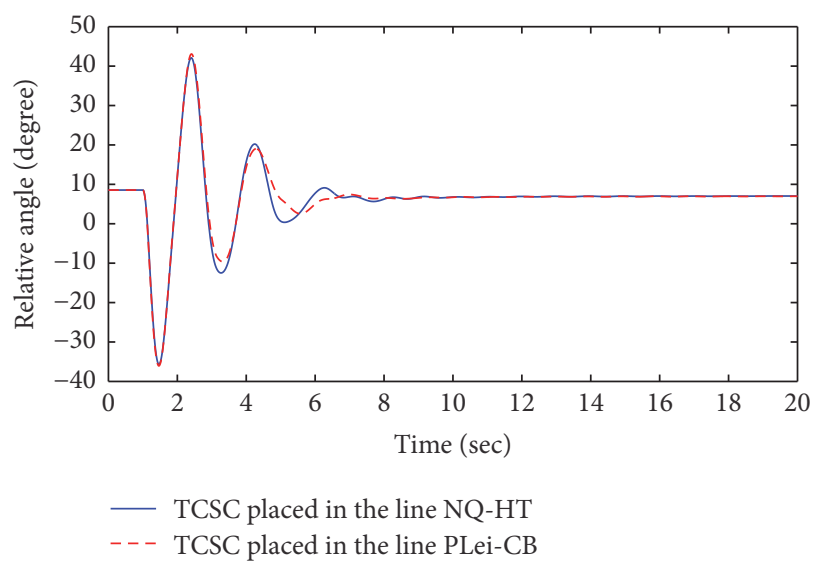

(b)

FIGURE 12: The relative angle oscillations of generator: (a) QuangNinh; (b) VinhTan.

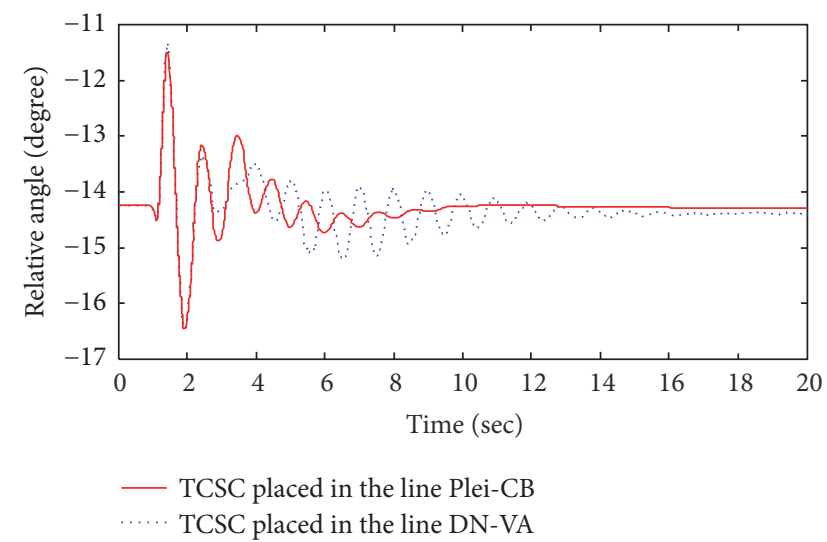

(a)

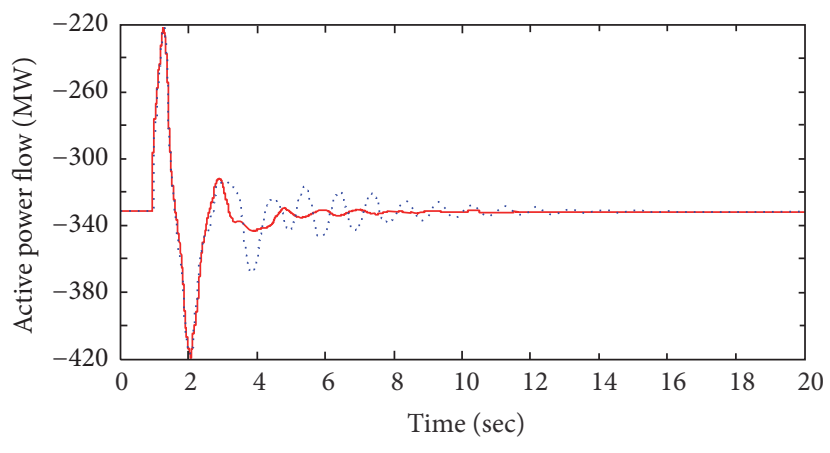

— TCSC placed in the line Plei-CB TCSC placed in the line DN-VA

(b)

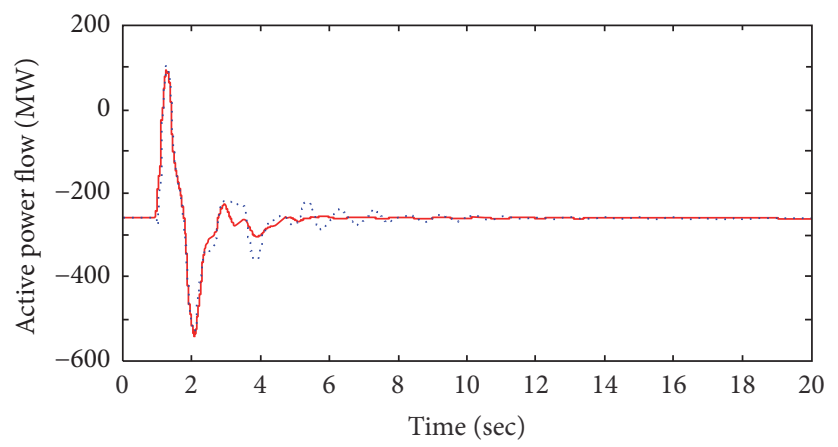

TCSC placed in the line Plei-CB

TCSC placed in the line DN-VA

(c)

FIGURE 13: The transient response: (a) the relative angle oscillations of generator SonLa; (b) the active power oscillations in the line NhoQuanSonLa; (c) the active power oscillations in the line HaTinh-VungAng.

fault location (in the line NQ-TT) compared with the TCSC placed at the Southern region (in the line PLei-CB).

Case 5. Also, for comparison of the other locations, the simulation for this case was done on scenario number 2 , supposing that the system has been operating at minimum load. Figure 13 plots the transient response of the system. For maximum load, the transferred power on the $500 \mathrm{kV}$ system from the North region to the South region is very high. Consequently, the amplitude deviation of the generator rotor 
angle and active power oscillations is very large. However, for minimum load, this deviation is negligible but it is still accordant with the proposed method. It could be seen from Figure 13 that the best effect is obtained in terms of oscillation damping of the generator rotor angle of SonLa and the active power in the lines of NhoQuan-SonLa and HaTinhVungAng.

\section{Conclusions}

In this paper, a relevant stochastic method for the optimal placement of TCSC controller has been presented to enhance the rotor angle stability and dampen the power system oscillations in the multimachine systems. This proposed method is developed from the energy approach based on the controllability and observability Gramian matrices of the linearized multimachine systems. The optimal placement depends on the trace indices of the Gramian matrices that have been calculated on the active power perturbation in the line of the network (for this study, the applied network is the Vietnamese $500 / 220 \mathrm{kV}$ power system).

The optimal placement for TCSC controller is determined based on the Gramian critical energy values that have been calculated on the small-signal stability analysis. However, the acquired results showed that the power system could operate perfectly under the influence of the transient conditions.

The time-domain simulation results on the transient stability analysis show that the rotor angle oscillations of generator and the power oscillations in the line are significantly dampened when the TCSC is placed in the line between PLeiku and CauBong, which has the maximum total energy value.

The Gramian-based reduction method has been also introduced for the purpose of reducing the calculation time of the Gramian critical energy when dealing with the largescale power systems.

\section{Appendix}

\section{Algorithm for the Transformation Matrix $M$}

First Step. Calculate the decomposition of the controllability Gramian:

$$
G_{c}=\mathbb{C}^{\mathrm{T}} \mathbb{C}
$$

where $\mathbb{C}$ is an upper triangular matrix, which has only zeros below the main diagonal, and such decomposition is called the Cholesky decomposition and can be implemented in MATLAB as function: $\mathbb{C}=\operatorname{chol}\left(G_{c}\right)$.

Then. Perform singular value decomposition for the product $\mathbb{C} G_{c} \mathbb{C}^{\mathrm{T}}$ :

$$
\mathbb{C} G_{c} \mathbb{C}^{\mathrm{T}}=U \Sigma U^{\mathrm{T}}
$$

where $U$ is orthogonal matrix and $\Sigma=\operatorname{diag}\left\{\sigma_{1}, \sigma_{2}, \sigma_{3}, \ldots, \sigma_{n}\right\}$ is the diagonal matrix.

In the End. The transformation matrix can be calculated as follows:

$$
M=\mathbb{C}^{\mathrm{T}} U^{\mathrm{T}} \Sigma^{-1 / 4}
$$

\section{Disclosure}

This paper is an applied and fully constituted version of the paper published in International Transactions on Electrical Energy Systems, 2016, Volume 26, Issue 7, pp. 1493-1510.

\section{Competing Interests}

The authors declare that there is no conflict of interests regarding the publication of this paper.

\section{Acknowledgments}

The authors sincerely acknowledge the financial support provided by Duy Tan University, Da Nang, Vietnam, Ton Duc Thang University, Ho Chi Minh, Vietnam, Industrial University of Ho Chi Minh City, Ho Chi Minh, Vietnam, and Quy Nhon University, Binh Dinh, Vietnam, for carrying out this work.

\section{References}

[1] L. L. Lai, H. T. Zhang, S. Mishra, D. Ramasubramanian, C. S. Lai, and F. Y. Xu, "Lessons learned from July 2012 Indian blackout," in Proceedings of the 9th IET International Conference on Advances in Power System Control, Operation and Management (APSCOM '12), pp. 1-6, IEEE, Hong Kong, November 2012.

[2] P. Kundur, Power System Stability and Control, McGraw-Hill, New York, NY, USA, 1994.

[3] G. Andersson, P. Donalek, R. Farmer et al., "Causes of the 2003 major grid blackouts in North America Europe, and recommended means to improve system dynamic performance," IEEE Transactions on Power Systems, vol. 20, no. 4, pp. 1922-1928, 2005.

[4] A. Gheorghe, M. Masera, M. Weijnen, and D. L. Vries, Critical Infrastructures at Risk, vol. 9, Kluwer Academic Publishers, Dordrecht, Netherlands, 2006.

[5] S. Larsson and E. Ek, "The black-out in southern Sweden and eastern Denmark, September 23, 2003," in Proceedings of the IEEE Power Engineering Society General Meeting, 2004.

[6] D. N. Kosterev, C. W. Taylor, and W. A. Mittelstadt, "Model validation for the august 10, 1996 wscc system outage," IEEE Transactions on Power Systems, vol. 14, no. 3, pp. 967-979, 1999.

[7] H. Nguyen-Duc, H. Cao-Duc, C. Nguyen-Dinh, and V. NguyenXuan-Hoang, "Simulation of a power grid blackout event in Vietnam," in Proceedings of the IEEE Power and Energy Society General Meeting (PESGM '15), p. 15, Denver, Colo, USA, July 2015.

[8] V. T. Dinh and H. H. Le, "Vietnamese $500 \mathrm{kV}$ power system and recent blackouts," in Proceedings of the IEEE Power and Energy Society General Meeting-Conversion and Delivery of Electrical Energy in the 21st Century, pp. 1-5, IEEE, Pittsburgh, Pa, USA, July 2008. 
[9] L. J. Cai, I. Erlich, and G. C. Stamtsis, "Optimal choice and allocation of FACTS devices in deregulated electricity market using genetic algorithms," in Proceedings of the IEEE PES Power Systems Conference and Exposition, pp. 201-207, New York, NY, USA, October 2004.

[10] H. Okamoto, A. Yokoyama, and Y. Sekine, "Stabilizing control of variable impedance power systems: applications to variable series capacitor systems," Electrical Engineering in Japan, vol. 113, no. 4, pp. 89-100, 1993.

[11] J. Ma, T. Wang, S. Wang et al., "Application of dual youla parameterization based adaptive wide-area damping control for power system oscillations," IEEE Transactions on Power Systems, vol. 29, no. 4, pp. 1602-1610, 2014.

[12] U. P. Mhaskar and A. M. Kulkarni, "Power oscillation damping using FACTS devices: modal controllability, observability in local signals, and location of transfer function zeros," IEEE Transactions on Power Systems, vol. 21, no. 1, pp. 285-294, 2006.

[13] N. Hingorani and L. Gyugyi, Understanding FACTS: Concepts and Technology of Flexible AC Transmission Systems, John Wiley \& Sons, Hoboken, NJ, USA, 2000.

[14] K. Tang and G. K. Venayagamoorthy, "Damping inter-area oscillations using virtual generator based power system stabilizer," Electric Power Systems Research, vol. 129, pp. 126-141, 2015.

[15] S. Gerbex, R. Cherkaoui, and A. J. Germond, "Optimal location of multi-type FACTS devices in a power system by means of genetic algorithms," IEEE Transactions on Power Systems, vol. 16, no. 3, pp. 537-544, 2001.

[16] B. Chaudhuri and B. C. Pal, "Robust damping of multiple swing modes employing global stabilizing signals with a TCSC," IEEE Transactions on Power Systems, vol. 19, no. 1, pp. 499-506, 2004.

[17] A. Rezaee Jordehi, "Particle swarm optimisation for dynamic optimisation problems: a review," Neural Computing and Applications, vol. 25, no. 7-8, pp. 1507-1516, 2014.

[18] A. Rezaee Jordehi and J. Jasni, "Parameter selection in particle swarm optimisation: a survey," Journal of Experimental \& Theoretical Artificial Intelligence, vol. 25, no. 4, pp. 527-542, 2013.

[19] E. Ghahremani and I. Kamwa, "Optimal placement of multipletype FACTS devices to maximize power system loadability using a generic graphical user interface," IEEE Transactions on Power Systems, vol. 28, no. 2, pp. 764-778, 2013.

[20] L. Rocha, R. Castro, and J. M. F. de Jesus, "An improved particle swarm optimization algorithm for optimal placement and sizing of STATCOM," International Transactions on Electrical Energy Systems, vol. 26, no. 4, pp. 825-840, 2015.

[21] R. Benabid, M. Boudour, and M. A. Abido, "Optimal location and setting of SVC and TCSC devices using non-dominated sorting particle swarm optimization," Electric Power Systems Research, vol. 79, no. 12, pp. 1668-1677, 2009.

[22] K. Ravi and M. Rajaram, "Optimal location of FACTS devices using improved particle swarm optimization," International Journal of Electrical Power \& Energy Systems, vol. 49, no. 1, pp. 333-338, 2013.

[23] M. Saravanan, S. M. R. Slochanal, P. Venkatesh, and J. P. S. Abraham, "Application of particle swarm optimization technique for optimal location of FACTS devices considering cost of installation and system loadability," Electric Power Systems Research, vol. 77, no. 3-4, pp. 276-283, 2007.

[24] D. P. Rini, S. M. Shamsuddin, and S. S. Yuhaniz, "Particle swarm optimization: technique, system and challenges," International Journal of Computer Applications, vol. 14, no. 1, pp. 19-26, 2011.
[25] L. Ippolito and P. Siano, "Selection of optimal number and location of thyristor-controlled phase shifters using genetic based algorithms," IEE Proceedings-Generation, Transmission and Distribution, vol. 151, no. 5, pp. 630-637, 2004.

[26] E. S. Ali and S. M. Abd-Elazim, "Stability improvement of multimachine power system via new coordinated design of PSSs and SVC," Complexity, vol. 21, no. 2, pp. 256-266, 2015.

[27] A. E. Dahej, S. Esmaeili, and A. Goroohi, "Optimal allocation of SVC and TCSC for improving voltage stability and reducing power system losses using hybrid binary genetic algorithm and particle swarm optimization," Canadian Journal on Electrical and Electronics Engineering, vol. 3, no. 3, pp. 100-107, 2012.

[28] A. R. Jordehi, "Brainstorm optimisation algorithm (BSOA): an efficient algorithm for finding optimal location and setting of FACTS devices in electric power systems," International Journal of Electrical Power \& Energy Systems, vol. 69, pp. 48-57, 2015.

[29] B. K. Kumar, S. N. Singh, and S. C. Srivastava, "Placement of FACTS controllers using modal controllability indices to damp out power system oscillations," IET Generation, Transmission \& Distribution, vol. 1, no. 2, article 209, 2007.

[30] P. S. Vaidya and V. P. Rajderkar, "Optimal location of series FACTS devices for enhancing power system security," in Proceedings of the 4th International Conference on Emerging Trends in Engineering and Technology (ICETET '11), pp. 185-190, Port Louis, Mauritius, November 2011.

[31] K. S. Verma, S. N. Singh, and H. O. Gupta, "FACTS devices location for enhancement of total transfer capability," in Proceedings of the IEEE Power Engineering Society Winter Meeting, vol. 3, pp. 522-527, Columbus, Ohio, USA, February 2001.

[32] L. Rouco and F. L. Pagola, "An eigenvalue sensitivity approach to location and controller design of controllable series capacitors for damping power system oscillations," IEEE Transactions on Power Systems, vol. 12, no. 4, pp. 1660-1666, 1997.

[33] A. Nasri, R. Eriksson, and M. Ghandhari, "Using trajectory sensitivity analysis to find suitable locations of series compensators for improving rotor angle stability," Electric Power Systems Research, vol. 111, pp. 1-8, 2014.

[34] V. Le, X. Li, Y. Li, Y. Cao, and C. Le, "Optimal placement of TCSC using controllability Gramian to damp power system oscillations," International Transactions on Electrical Energy Systems, vol. 26, no. 7, pp. 1493-1510, 2015.

[35] L. Van Dai, X. Li, P. Li, and L. C. Quyen, "An optimal location of static VAr compensator based on Gramian critical energy for damping oscillations in power systems," IEEJ Transactions on Electrical and Electronic Engineering, vol. 11, no. 5, pp. 577-585, 2016.

[36] K. Glover, "All optimal Hankel-norm approximations of linear multivariable systems and their $\mathrm{L}^{\infty}$-error bounds," International Journal of Control, vol. 39, no. 6, pp. 1115-1193, 1984.

[37] S. N. Singh and A. K. David, "A new approach for placement of FACTS devices in open power markets," IEEE Power Engineering Review, vol. 21, no. 9, pp. 58-60, 2001.

[38] M. A. Abido, "Pole placement technique for PSS and TCSCbased stabilizer design using simulated annealing," International Journal of Electrical Power \& Energy System, vol. 22, no. 8, pp. 543-554, 2000.

[39] Prime Minister of Vietnam, "Approval of the National Master Plan for power development for the 2011-2020 period with visions extended 2030," Decision 428/QĐ-TTG, 2016.

[40] P. W. Sauer and M. A. Pai, Power System Dynamics and Stability, Prentice-Hall, Upper Saddle River, NJ, USA, 1998. 
[41] D. Chatterjee and A. Ghosh, "Transient stability assessment of power systems containing series and shunt compensators," IEEE Transactions on Power Systems, vol. 22, no. 3, pp. 1210-1220, 2007.

[42] A. B. Leirbukt, "Damping control design based on time-domain identified models," IEEE Transactions on Power Systems, vol. 14, no. 1, pp. 172-178, 1999.

[43] S. Nagalakshmi and N. Kamaraj, "Secured loadability enhancement with TCSC in transmission system using computational intelligence techniques for pool and hybrid model," Applied Soft Computing Journal, vol. 11, no. 8, pp. 4748-4756, 2011.

[44] C. R. Fuerte-Esquivel, E. Acha, and H. Ambriz-Pérez, "A thyristor controlled series compensator model for the power flow solution of practical power networks," IEEE Transactions on Power Systems, vol. 15, no. 1, pp. 58-64, 2000.

[45] Y. Xiao, Y. H. Song, and Y. Z. Sun, "Power flow control approach to power systems with embedded FACTS devices," IEEE Transactions on Power Systems, vol. 17, no. 4, pp. 943-950, 2002.

[46] D. Georges, "Use of observability and controllability gramians or functions for optimal sensor and actuator location in finitedimensional systems," in Proceedings of the 34th IEEE Conference on Decision and Control, pp. 3319-3324, December 1995.

[47] G. Obinata and B. D. O. Anderson, Model Reduction for Control System Design, Springer, London, UK, 2001.

[48] M. A. Wicks and R. A. DeCarlo, "An energy approach to controllability," in Proceedings of the 27th IEEE Conference on Decision and Control, pp. 2072-2077, December 1988.

[49] A. Ishchenko, J. M. A. Myrzik, and W. L. Kling, "Dynamic equivalencing of distribution networks with dispersed generation using Hankel norm approximation," IET Generation, Transmission \& Distribution, vol. 1, no. 5, pp. 818-825, 2007.

[50] Siemens PTI. PSS/E 30.2 Program Operational Manual, vol. 2, 2005.

[51] H. Shayeghi, H. A. Shayanfar, S. Jalilzadeh, and A. Safari, "TCSC robust damping controller design based on particle swarm optimization for a multi-machine power system," Energy Conversion and Management, vol. 51, no. 10, pp. 1873-1882, 2010. 


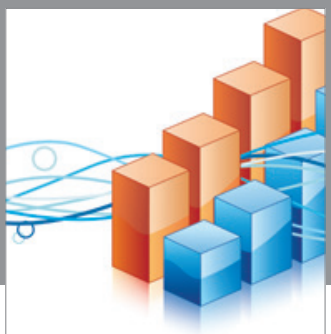

Advances in

Operations Research

vatem alat4

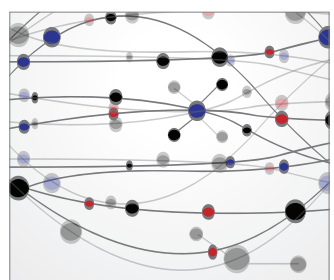

\section{The Scientific} World Journal
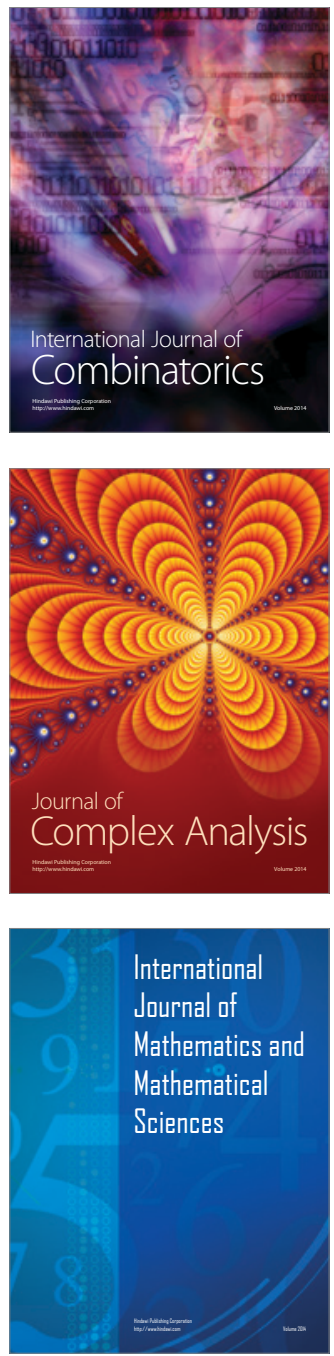
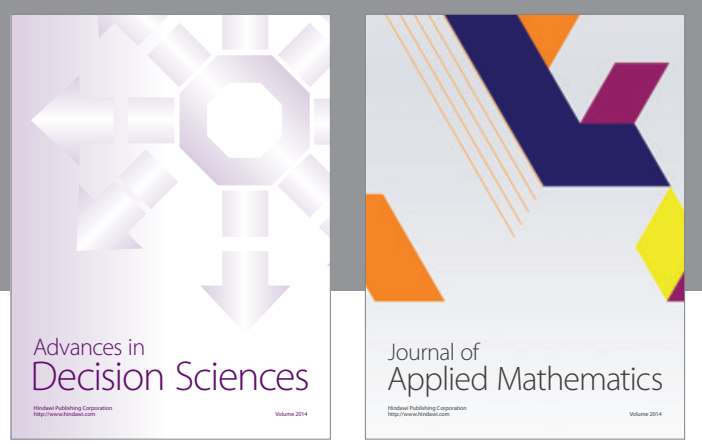

Algebra

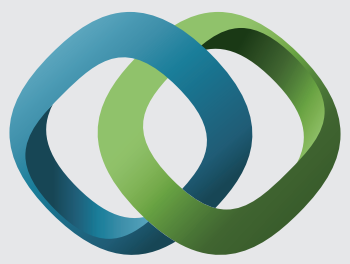

\section{Hindawi}

Submit your manuscripts at

https://www.hindawi.com
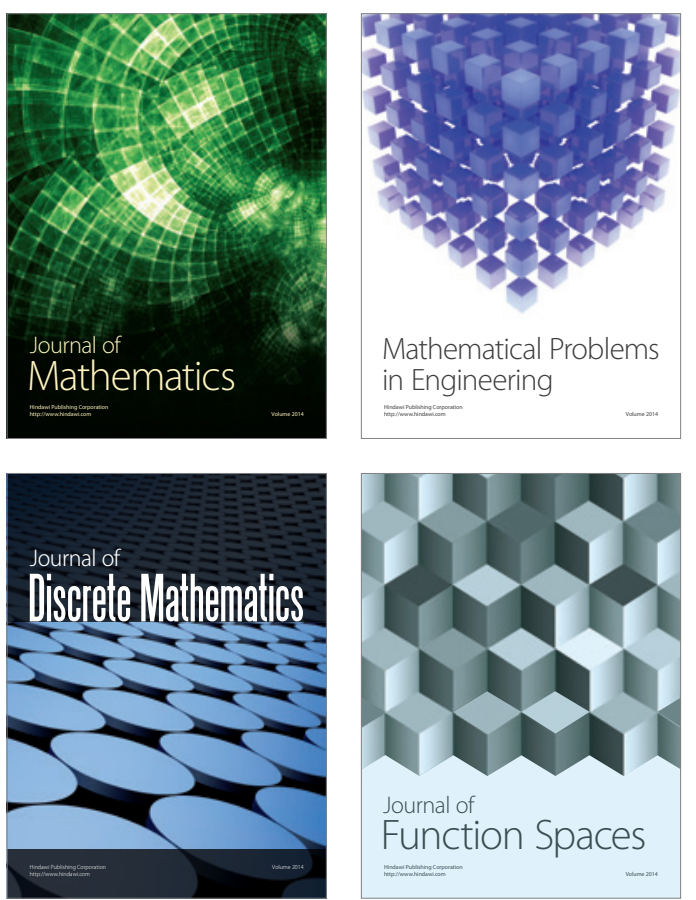

Mathematical Problems in Engineering
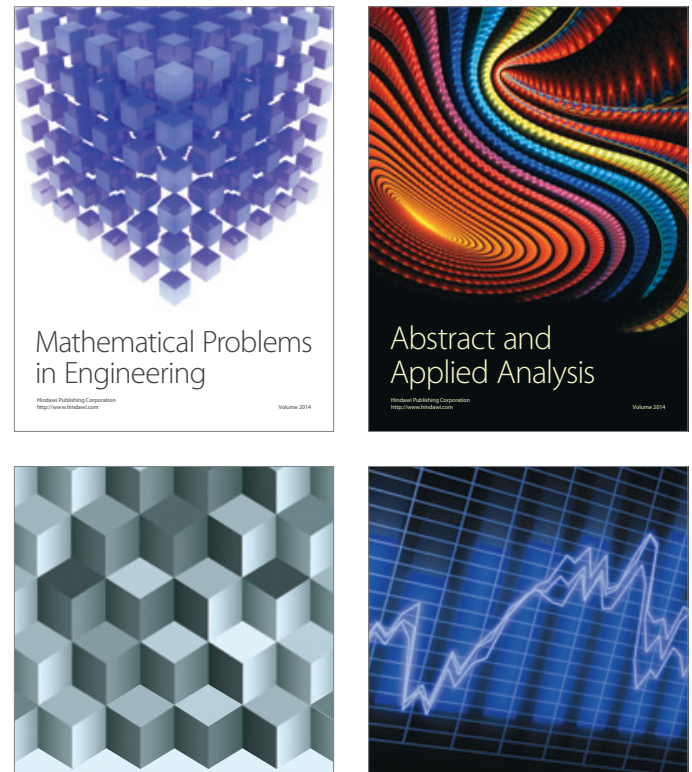

Journal of

Function Spaces

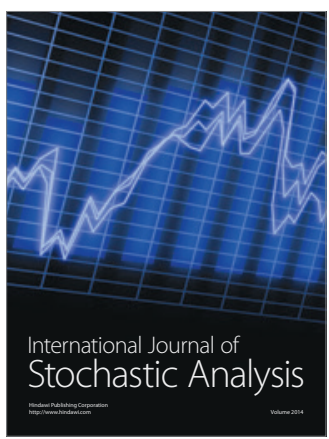

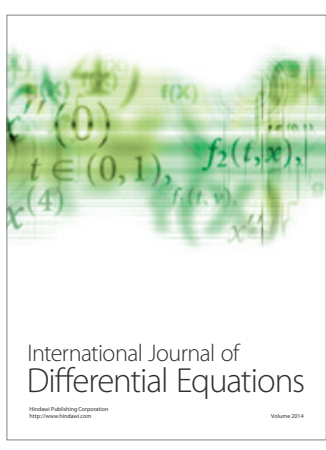
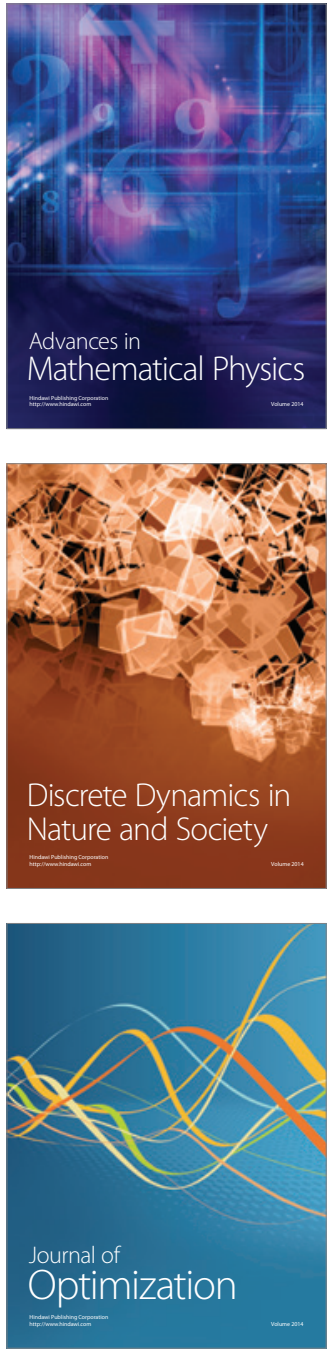